\title{
Disorder in Charge Transport in Doped Polymers**
}

\section{By Mark Van der Auweraer,* Frans C. De Schryver,* Paul M. Borsenberger, and Heinz Bässler}

\section{Introduction}

The interest in the transport of charge carriers in molecularly doped polymers and related materials is related to the important application of these systems as photoreceptors in xerography ${ }^{[1-3]}$ and to the theoretical challenge of understanding the charge transport in these disordered solids. Charge carrier propagation in disordered media containing a charge transport agent $\mathrm{D}$ can be described as a sequence of one-electron oxidation-reduction processes, where each of the electron or hole transfer steps is assumed to be completely independent of the preceding one (incoherent transport). For hole transport, some groups are initially positively charged (radical cations). Under an applied field, neutral molecules will transfer electrons to the radical cations. For this to occur, the groups must be donor-like in their neutral state. For electron transport, electrons are displaced from the anion radicals to neutral molecules, which must be acceptor-like in their neutral state.

Those transport processes can be investigated using a xerographic technique,${ }^{[4,5]}$ where the photo-induced discharge of a sample charged by a corona is determined, or by the determination of transient photocurrents. The latter method, ${ }^{[6-8]}$ although requiring a more elaborate setup, is able to yield independent information on the quantum yield of photogeneration, the mobility of the charge carriers, and the nature of the charge transport process. In this method the sample is sandwiched between a semi-transparent top electrode and a counter electrode. A strongly absorbed light pulse impinging through the top electrode creates a sheet of charge carriers

[*] Prof. M. Van der Auweraer, Prof. F. C. De Schryver Laboratory for Molecular Dynamics and Spectroscopy K. U. Leuven

Celestijnenlaan 200-F, B-3001 Heverlee (Belgium)

Dr. P. Borsenberger

Office Imaging Division, Eastman Kodak Company

Rochester, NY 14650-01714 (USA)

Prof. H. Bässler

Fachbereich Physikalische Chemie, Philipps-Universität

Hans-Meerwein-Strasse, D-35032 Marburg (FRG)

[**] M. V.d. A. is a "Onderzoeksleider" of the Belgian "Fonds voor Kollektief Fundamenteel Onderzoek". The authors thank Agfa-Gevaert N. V. for financial support. The continuing support of the Belgian "Fonds voor Kollektief Fundamenteel Onderzoek" and the Ministry of Science Programming through IUAP 1II-040 and IUAP II-16 is gratefully acknowledged. The authors are indebted to D. Terrell (Agfa-Gevaert) for the sample of pEFTP. H. B. thanks the "Deutsche Forschungsgemeinschaft" and the "Fond der Chemischen Industrie". that migrates through the sample under the influence of the applied field (Fig. 1). Depending upon the sign of the applied potential difference and the transport material, this method allows charge transport by either electrons or holes to be investigated. ${ }^{[6-10]}$

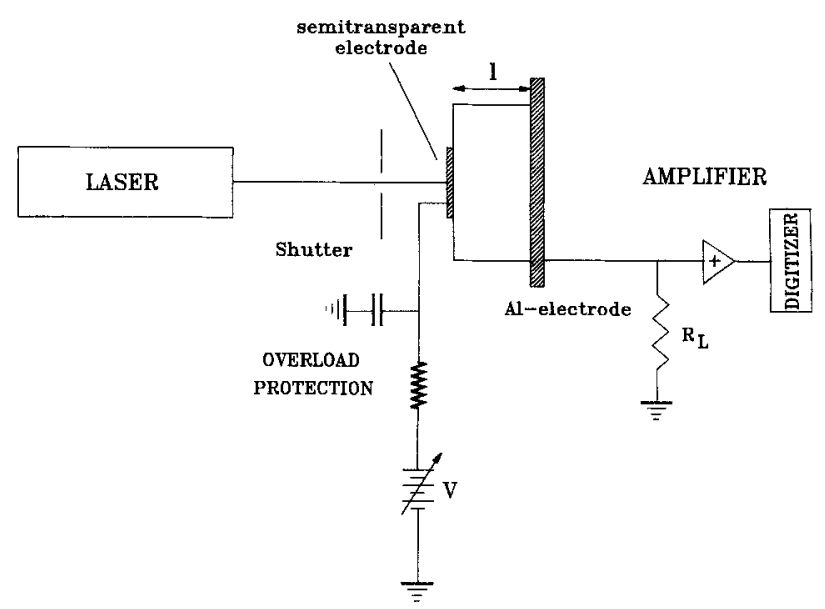

Fig. 1. Setup for the determination of transient photocurrents $[6,7]$.

For a straightforward interpretation of the transient photocurrents the electrodes should be non-injecting and hence create a blocking contact between the top electrode and the sample to avoid large dark currents and the creation of a space charge. The counter electrode should allow an efficient drain of the charge carriers that have crossed the sample to the electrode. ${ }^{[11,12]}$ The energy of the light pulse should be such that the photogenerated charge is much smaller than the product of the applied voltage and the capacitance of the illuminated area of the sample. Otherwise the features of the transient photocurrent will be distorted by the space charge. ${ }^{[13,14]}$ The investigation of transient photocurrents was originally ${ }^{[15,16]}$ developed for inorganic materials and later applied ${ }^{[6,7]}$ to organic single crystals, which are characterized by a non-dispersive charge transport. In this case the transient photocurrent has essentially a rectangular shape. ${ }^{[6,7]}$ The time dependence of the current can be described by Equations 1a and $b$, where $t_{\mathrm{tr}}$ and $q$ correspond to the transit times of the charge carriers $[\mathrm{s}]$ and the total photogenerated charge $[\mathrm{C}]$, respec- 
tively. The transit time $t_{\mathrm{tr}}$ is related to the mobility $\mu$ $\left[\mathrm{cm}^{2} \mathrm{~V}^{-1} \mathrm{~s}^{-1}\right]$ by Equation 2 .

$$
\begin{aligned}
& i(t)=\frac{q}{t_{\mathrm{tr}}} \text { for } t<t_{\mathrm{tr}} \\
& i(t)=0 \text { for } t>t_{\mathrm{tr}} \\
& t_{\mathrm{tr}}=\frac{l}{v_{\mathrm{dr}}}=\frac{l}{\mu E}=\frac{l^{2}}{\mu V}
\end{aligned}
$$

In Equation 2, $l, v_{\mathrm{dr}}, E$ and $V$ correspond to the thickness of the sample $[\mathrm{cm}]$, the drift velocity $\left[\mathrm{cm} \mathrm{s}^{-1}\right]$, the applied field $\left[\mathrm{Vcm}^{-1}\right]$, and the applied potential difference $[V]$, respectively. For non-dispersive transport the spread $\left\langle\Delta x^{2}\right\rangle^{1 / 2}$ of a sheet of charge carriers that has traveled a distance $x$ is given $^{[17,18]}$ by Equation 3, which can be written as Equation 4 with Equation 5.

$$
\begin{aligned}
& \frac{\left\langle\Delta x^{2}\right\rangle^{1 / 2}}{x}=\frac{k T}{e E}\left(\frac{2}{D t}\right)^{1 / 2} \\
& \left\langle\Delta x^{2}\right\rangle^{1 / 2}=(2 D t)^{1 / 2} \\
& e D=\mu k T
\end{aligned}
$$

In Equations 3-5, $e$ and $D$ correspond to the charge of an electron or a hole and the diffusion coefficient of the charge carriers, respectively. Equations 3-5 imply the validity of Einstein's law relating mobility to diffusivity. ${ }^{[18]}$ Application of Equations 1-5 assumes that no photogenerated carriers are lost by recombination. Those recombination processes were investigated using "interrupt-field time-of-flight photoconductivity". ${ }^{[19]}$ In this method the electric field is switched off immediately after the generation of the charge carriers and a large collecting field is applied after a delay time to collect the remaining charge carriers. However, the extent to which the recombination rates of the charge carriers obtained using this method are relevant for the experimental conditions in a normal time-of-flight experiment remains uncertain.

While Equations $1-5$ are able to describe transient photocurrents in organic and inorganic single crystals, ${ }^{[5,6,15,16,20]}$ the transient photocurrents observed for several amorphous inorganic $^{[21]}$ and organic systems ${ }^{[22]}$ showed a continual decrease of the photocurrent. Often plotting the logarithm of the current versus the logarithm of the time yields plots intersecting at $t=t_{\mathrm{rr}}{ }^{[17,23-25]}$ (Eq. 6). For photocurrents obtained at different applied fields and temperatures it was furthermore often possible to superpose plots of $\ln \left[i(t) / i\left(t_{\mathrm{tr}}\right)\right]$. This property is called universality. ${ }^{[17]}$

$$
\begin{aligned}
& i(t) \propto t^{\alpha-1} \text { for } t<t_{\mathrm{tr}} \\
& i(t) \propto i\left(t_{\mathrm{tr}}\right) t^{\beta-1} \text { for } t>t_{\mathrm{tr}}
\end{aligned}
$$
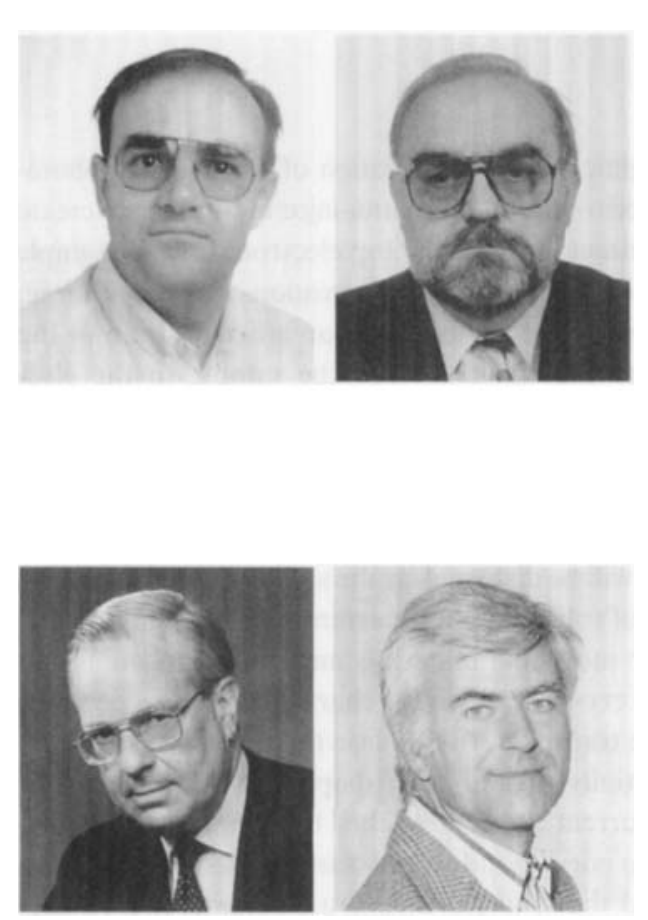

Mark Van der Auweraer received a Ph.D. degree in chemistry from the $K . U$. Leuven in 1981. After post-doctoral work with an Alexander von Humboldt fellowship at the Fritz-Haber-Institut (Berlin), he returned to K. U. Leuven, where he habilitated in 1990. He was awarded the Grammaticakis-Neumann Prize in 1992. His research interests are focused on electron and excitation transfer in organized systems and photconduction.

Frans C. De Schryver was awarded his Ph.D by the K. U. Leuven in 1964 and after two year's post-doctoral research as a Fullbright Fellow with Prof. C. S. Marvel at the University of Arizona, Tucson, returned to Leuven, where he became full professor in 1975. His research interests are the photochemistry and photophysics of organic molecules with emphasis on macromolecules and supramolectlar structures. He was recently awarded a Humboldt Research Prize.

Paul M. Borsenberger, originally from St. Louis, received a B.S. in metallurgy from the Missouri School of Mines and Metallurgy. Following three years in the U.S. Army, he earned M.S. and Ph.D. degrees in materials science at Stanford University. In 1967 he joined the Research Laboratories of Eastman Kodak Company. Since then his research interests have been related to xerography and photoconductivity of disordered solids.

Heinz Bässler is professor of physical chemistry at the Department ("Fachbereich") of Physical Chemistry at Philipps-Universität in Marburg, FRG. He received his Ph.D. in physics from the Technical University in Munich in 1963. After a post-doctoral year in Philadelphia, he moved to his present position in 1970. His research interests include electro-optic properties of organic solids. 
with

$0<\alpha<1$ and $\beta<0$

These features of the transient photocurrents suggested a dispersive transport where the velocity of a sheet of carriers decreases as the packet traverses the sample. This is accompanied by an increase in the width of the sheet of charge carriers that is proportional to $t$ rather than $t^{1 / 2}$.

$\left\langle\Delta x^{2}\right\rangle^{1 / 2} \propto t$

Hence the mean arrival time of the carriers becomes an ill-defined quantity which no longer allows the determination of mobility in an unambiguous way using Equation 2. Seki ${ }^{[26]}$ and Scher and Montroll ${ }^{[1]}$ attributed the occurrence of dispersive transport to a hopping mechanism with a waiting time distribution $\psi(t)$ that is characterized by infinite moments. For hopping between isoenergetic sites with a random positional distribution, " $\mathrm{r}$ hopping", the waiting time distribution can be approximated by Equation 8 if the hopping probability between two sites separated by a distance $a$ is proportional to $\exp (-a \gamma)$. As $\alpha$ depends only to a minor extent on $t$, Equations 810 can describe a transient photocurrent and correspond to Equation 6 with $\alpha=-\beta$.

$\psi(t) \propto t^{-(1+\alpha)}$

with

$\alpha=-\beta=\frac{1}{3} \eta\left[\ln \left(t / t_{\mathrm{tr}}\right)\right]^{2}$

and

$\eta=1 /(\gamma a)^{3}$

According to Equations 6-10 the relationships 11 and 12 hold between the transit time $t_{\mathrm{tr}}$, the mobility $\mu$, and the thickness $l$ of the sample and the applied field $E$.

$t_{\mathrm{tr}} \propto(l / E)^{1 / \alpha}$

$\mu \propto(l / E)^{(1-1 / \alpha)}$

According to Equations 9 and 10 small values of $a$, corresponding to a high concentration of the transport molecules, will yield a large value of $\eta$ and hence of $\alpha$, corresponding to a nearly non-dispersive transport, while large values of $a$, corresponding to a low concentration, will yield values of $\alpha$ close to zero, corresponding to a highly dispersive transport. ${ }^{[23-25]}$ For non-dispersive transport, the waiting time distribution would be characterized by finite moments $\left(\int \psi(t) t^{n} \mathrm{~d} t \neq \infty\right)$. This could be realized by a waiting time distribution $\psi(t)$ resembling Equation 13.

$\psi(t) \propto \exp (-t / \tau)$

Although the analysis of Scher and Montroll could explain some aspects of charge transport in disordered systems success- fully, the observed values of $\alpha$ often differed from those observed for $\beta .^{[27-29]}$ Furthermore, for several systems, deviations from universality were observed when a system was evaluated at different temperatures. ${ }^{[27-32]}$ Those deviations could be attributed to the energetic disorder between the different sites involved in the hopping process. Beside fluctuations in the intermolecular distance and mutual orientation of two molecules (off-diagonal disorder) ${ }^{[33]}$ an amorphous system will always be characterized by fluctuations of the molecular energy levels (diagonal disorder). Due to these fluctuations the narrow transport bands of molecular crystals are split into a distribution of completely localized states between which hopping occurs. The inhomogeneous broadening of the absorption and fluorescence spectra of organic glasses or of molecular dispersions of chromophores in polymers as opposed to crystalline counterpart structures are attributed to these disorder effects. It is easy to understand that for positively or negatively charged radical ions this diagonal disorder is brought about by fluctuations of the van der Waals interaction energy or of the ion-induced dipole or of the ionpermanent dipole interactions. ${ }^{[34,35]}$ Starting from an exponential (Eq. 14) or a Gaussian distribution (Eq. 15) of the density of states (DOS), it is possible to use Monte Carlo calculations to describe the average position and energy of a charge carrier that is injected into this distribution of energy levels at $t=0 .{ }^{[36,37]}$

$\rho(\varepsilon)=\varepsilon_{\mathrm{s}} \exp \left(-\frac{\varepsilon}{\varepsilon_{\mathrm{s}}}\right)$ for $\varepsilon>0$

$\rho(\varepsilon)=\frac{1}{2 \pi \sigma^{2}} \exp \left(-\frac{\varepsilon^{2}}{2 \sigma^{2}}\right)$

In the framework of this model, off-diagonal disorder can be introduced as an overlap parameter $\Gamma_{i j}$ which describes the interaction between the HOMOs (highest occupied molecular orbitals) and the LUMOs (lowest unoccupied molecular orbitals) of sites $i$ and $j . \Gamma_{i j}$ can be given by the sum of the parameters $\Gamma_{i}$ and $\Gamma_{j}$, which are randomly distributed with mean $\gamma a$ and variance $\Sigma / \sqrt{2}$. In this model the rate $v_{i j}$ for a jump between site $i$ and $j$ is given by Equation 16 .

$$
\begin{aligned}
& v_{i j}=v_{0} \exp \left(-\gamma \Delta R_{i j}\right) \exp \left[-\left(\frac{\varepsilon_{j}-\varepsilon_{i}}{k T}\right)\right] \text { for } \varepsilon_{j}>\varepsilon_{i} \\
& v_{i j}=v_{0} \exp \left(-\gamma \Delta R_{i j}\right) \text { for } \varepsilon_{j}<\varepsilon_{i}
\end{aligned}
$$

In Equation 16, $v_{0}$ and $\Delta R_{i j}$ correspond to an electronic prefactor $\left[\mathrm{s}^{-1}\right]$ and the center-to-center distance between two sites $i$ and $j[\AA]$, respectively. This distance is characterized by an average value $a$. Hence relative variation of the electronic intersite coupling is given by $\Sigma /(2 \gamma a)$. Hopping in an exponential distribution ${ }^{[38]}$ of energy levels can be used to describe the dispersive transport of charge carriers in inorganic amorphous materials or polyvinylcarbazole. In the latter materials the charge carrier transport can also be described in a multiple trapping formalism (see below). This 
formalism was however less suitable for describing the transport of holes by molecules dispersed in an inert polymer. At higher temperatures the transient photocurrents are always characterized by a fast initial decrease followed by a plateau, until at $t=t_{\mathrm{tr}}$ the current photocurrent decays to zero.

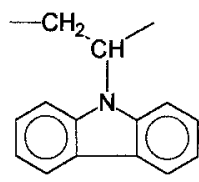

PVK

Although the plateau suggests a non-dispersive transport ( $\alpha$ approaches 1 in Equation 6a), the tail of the photocurrent, corresponding to the spread of the sheet of charge carriers $\left(\left\langle\Delta x^{2}\right\rangle^{1 / 2} / \mu E\right)$ is several orders of magnitude wider than expected for non-dispersive transport. Simulations starting from a Gaussian DOS were, however, able to simulate the features of the transient photocurrents. ${ }^{[36-40]}$ The fundamental difference between hopping in an exponential DOS and in a Gaussian DOS is that while in the former system the average energy of the hopping carriers $\langle\varepsilon\rangle$ relaxes to $-\infty$ it relaxes to $-\sigma^{2} /(k T)$ in the latter system. Therefore Equation $6 \mathrm{a}$ is no longer adequate to describe the hopping in a Gaussian DOS at times shorter than $t_{\mathrm{tr}}$. On a heuristic basis, Scott et al. ${ }^{[41]}$ therefore derived Equation 17, based on combination of the slowing down of the charge carriers due to the relaxation of the energy in a DOS and a Gaussian distribution of inverse transit times. Here $t_{0}$ and $\sigma_{1}$ are the average

$i(t)=A t^{\alpha-1}\left[1-\int_{0}^{t} \frac{1}{\sigma_{1} t^{\prime 2}(2 \pi)^{1 / 2}} \exp \left(-\frac{\left(1 / t^{\prime}-1 / t_{0}\right)^{2}}{2 \sigma_{\mathrm{I}}^{2}}\right) \mathrm{d} t^{\prime}\right]$

and the standard deviation of the inverse transit times, respectively. Unless $\alpha<1$, this expression does not become zero when $t \rightarrow \infty .{ }^{[42]}$ This means that $\alpha$ not only governs the short time but also the long time behavior, and this could be one of the reasons for the correlation between $\alpha$ and $\sigma_{1}$ suggested by Scott. ${ }^{[41]}$ As long as $t_{0}>1 / \sigma_{\mathrm{I}}$, Equation 17 will be very close to zero at times much larger than $t_{0}$. However, when $1 / \sigma_{1}$ becomes large compared to $t_{0}$, which will occur for more dispersive transport, the long time behavior of Equation 17 could be improved by replacing ${ }^{[41]}$ Equation 17 by Equation 18, where erf is the error function.

$i(t)=\frac{1}{2} A t^{x-1}\left[\operatorname{erf}\left(\frac{1 / t_{0}}{\sigma_{1} \sqrt{2}}\right)+\operatorname{erf}\left(\frac{1 / t-1 / t_{0}}{\sigma_{1} \sqrt{2}}\right)\right]$

Contrary to Equation 17, Equation 18 does become zero for $t \rightarrow \infty$. The transit times obtained by fitting experimental results to Equation 17 or 18 permit the computational determination of a mean carrier mobility in a true sense. Generally, the mobility obtained using Equation 17 or 18 and those derived from the transit times determined via the intersection of asymptotes of Equations $6 \mathrm{a}$ and $6 \mathrm{~b}$ indicate that the latter model always yields smaller values of the transit time. While $t_{\mathrm{tr}}$ corresponds to the arrival of the fastest carriers at the opposite electrode, $t_{0}$ corresponds to the most probable transit time.

\section{Temporal Features of the Photocurrents}

Figure 2 shows typical features of a transient photocurrent obtained by Monte Carlo calculations. On increasing $\sigma /(k T)$

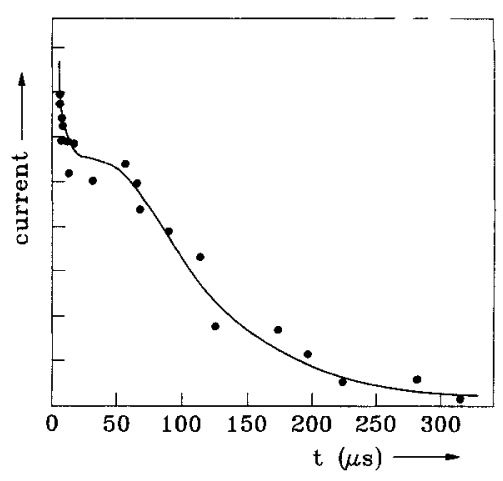

Fig. 2. Comparison of an experimental photocurrent transient (-) at $T=$ $312 \mathrm{~K}$ for a sample of 75\% 1,1-bis(di-4-tolylaminophenyl)cyclohexane (TAPC) in polycarbonate (PC) with data simulated for $\sigma=3.5 k T$ and $\Sigma=3.25$ [39].

from 1.4 to 4.0 , a gradual transition from a non-dispersive photocurrent transient to a dispersive photocurrent transient is observed. A typical experimental transient trace, shown in Figure 2 for a sample containing $75 \%$ of 1,1-bis(di-4-tolylaminophenyl)cyclohexane (TAPC) in polycarbonate (PC) at $312 \mathrm{~K}$, can be fitted to a simulated photocurrent transient, ${ }^{[37,39]}$ with $\sigma=3.50 \mathrm{kT}$ and $\Sigma=3.25$. The photocurrent transient re-<smiles>Cc1ccc(N(c2ccc(C)cc2)c2ccc(C3(c4ccc(N(c5ccc(C)cc5)c5ccc(C)cc5)cc4)CCCCC3)cc2)cc1</smiles>

ported in Figure 2 is typical for a large range of materials, temperatures, and applied field strengths. Similar photocurrent transients are obtained for aromatic donors dispersed in polymers and thin vapor-deposited layers of the same molecules. ${ }^{[30,33,39-4]}$ Although it is often impossible to analyze the photocurrent transients in the framework of Equation 6, they can be fitted to Equation 17 or 18 for a large range of materials and matrices ${ }^{[41-46]}$ (Fig. 3). For hole transport materials, fitting to Equation 17 or 18 generally yields values of $\alpha$, $\sigma_{1} t_{0}$ and $t_{0}$ that are identical to within the experimental error. 
photocurrent ( a.u. )

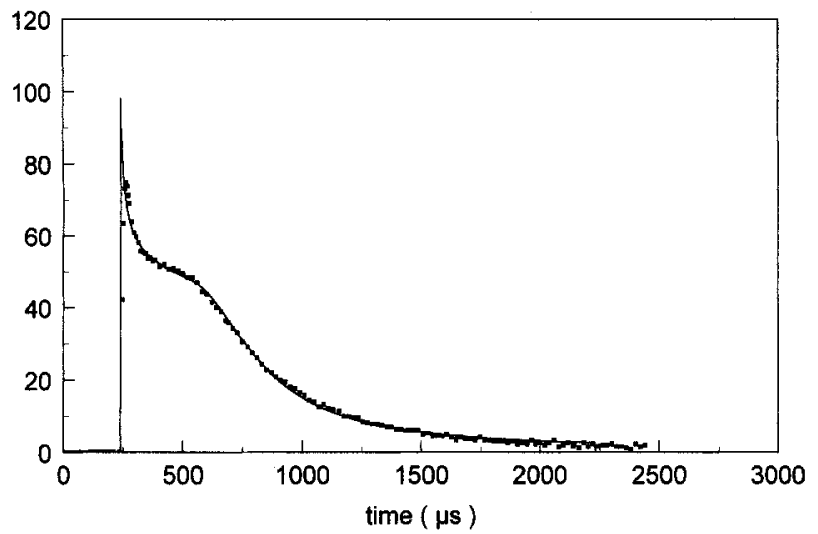

Fig. 3. Analysis of the transient photocurrent obtained for a $24 \%$ pEFTP in polycarbonate at $298 \mathrm{~K}$ and at an applied voltage of $1300 \mathrm{~V}$ in the framework of Equation 17 [42]. The sample thickness was $12.7 \mu \mathrm{m}$. Excitation occurred at $320 \mathrm{~nm}$. Analysis in the framework of Equation 18 yielded identical results, $\mathbf{n}$, experimental data; - - calculated decay.

A systematic investigation of the influence of the applied field on the fit parameters obtained for [4-[bis(4-ethylphenyl)amino]phenyl]- $N, N, N^{\prime}, N^{\prime}$-tetrakis(4-ethylphenyl)-[1,1': $3^{\prime}, 1^{\prime \prime}$-terphenyl]-4,4"-diamine (pEFTP) dispersed in polycarbonate<smiles>CCc1ccc(N(c2ccc(C)cc2)c2ccc(-c3cc(-c4ccc(N(c5ccc(C)cc5)c5ccc(C)cc5)cc4)cc(-c4ccc(N(c5ccc(C)cc5)c5ccc(CC)cc5)cc4)c3)cc2)cc1</smiles>

suggests ${ }^{[42]}$ that upon increasing the applied field $\sigma_{1} t_{0}$ increases from $0.35 \pm 0.1$ at low fields $\left(9.0 \times 10^{4} \mathrm{Vcm}^{-1}\right)$ to values ranging from 0.3 to 0.7 at high fields $\left(0.8\right.$ to $\left.1.6 \times 10^{6} \mathrm{Vcm}^{-1}\right)$ depending upon the temperature and the concentration of pEFTP (Fig. 4). While at low applied fields $\sigma_{1} t_{0}$ does not depend upon the temperature or the concentration, it increases at high fields upon decreasing the temperature or the concentration. The values of $\alpha$ increase under all conditions upon increasing the temperature. While the values of $\alpha$ at low fields do not depend upon the concentration, the values at high fields increase upon increasing the concentration (Fig. 5). This parallelism between the trends observed for $\alpha$ and $\sigma_{1} t_{0}$ corroborate the correlation between $\alpha$ and $\sigma_{\mathrm{I}} t_{0}$ suggested by Scott for

\section{$\sigma \cdot$ TTR}

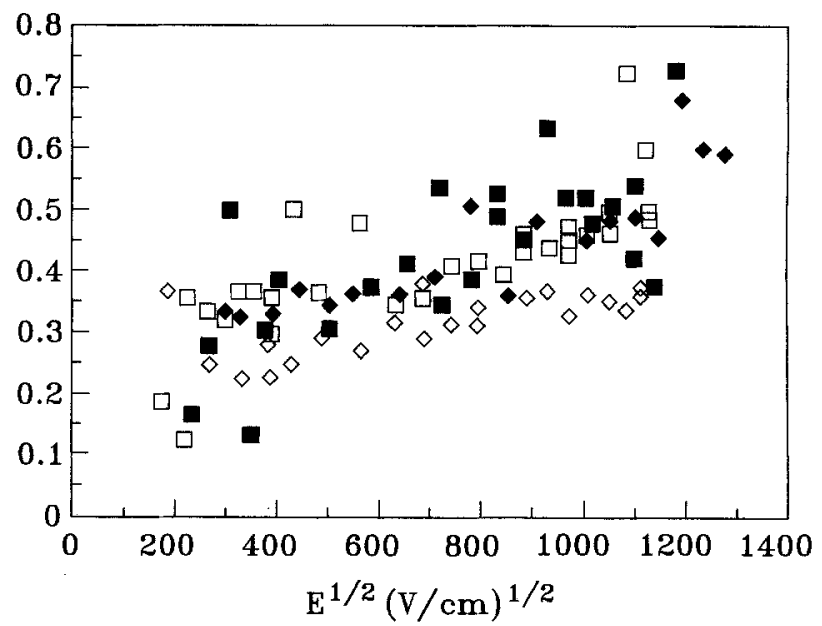

Fig. 4. Dependence of $\sigma_{\mathbf{l}} t_{0}$ on the applied field for pEFTP dispersed in polycarbonate. $\square, 24$ wt- $\%$ at $298 \mathrm{~K} ; \diamond, 24$ wt- $\%$ at $343 \mathrm{~K}$; $=11$ wt- $\%$ at $298 \mathrm{~K}$; • , $11 \mathrm{wt} \%$ at $343 \mathrm{~K}[42]$.

p-diethylaminobenzaldehyde-diphenylhydrazone (DEH).$^{[41]}$ The more dispersive character of the transport upon increasing the applied field is on first sight surprising. It is, however, also predicted by simulations. ${ }^{[37]}$

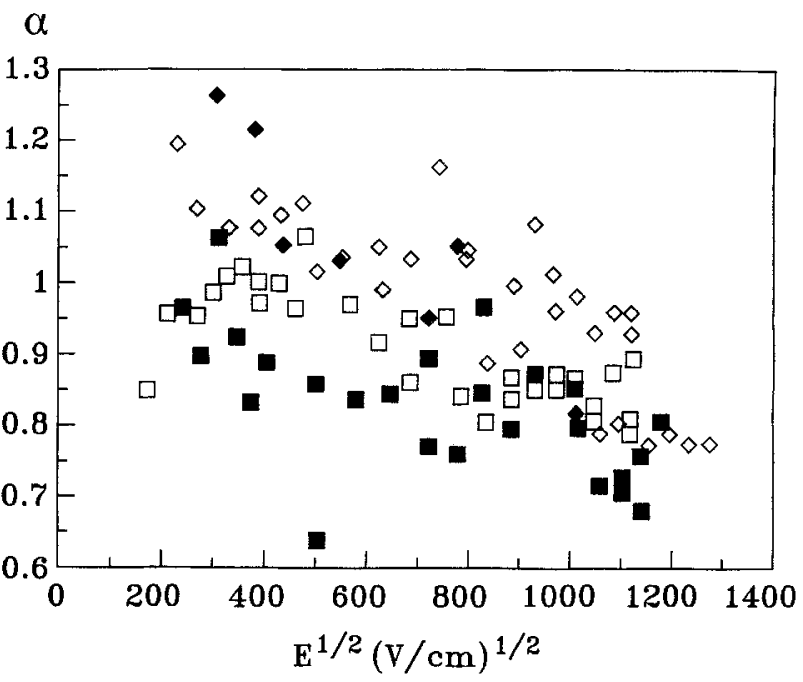

Fig. 5. Dependence of $\alpha$ on the applied field for pEFTP dispersed in polycarbonate. $\mathbf{\square} 24 \mathrm{wt}-\%$ at $298 \mathrm{~K} ; \diamond, 24 \mathrm{wt}-\%$ at $343 \mathrm{~K}$;, $11 \mathrm{wt}-\%$ at $298 \mathrm{~K}$; • $11 \mathrm{wt}-\%$ at $343 \mathrm{~K}[42]$.

The quantitative analysis of the features of the transient photocurrents and their field dependence suggests that amorphous $\mathrm{TAPC}^{[30,39]}$ or $\mathrm{DEH}^{[48]}$ and TAPC, ${ }^{[39]} \mathrm{DEH}^{[41,49,50]}$ tritolylamine (TTA) ${ }^{[43,44,51]}$ and pEFTP $^{[45,46]}$ dispersed in polycarbonate give rise to a hole transport that can be explained by models based on the hopping in a Gaussian DOS. A similar result was obtained for several electron transporting molecules such as a $4: 1$ mixture of $3,3^{\prime}$-dimethyl-5, $5^{\prime}$-di-tert-butyl 
diphenoquinone (33DPQ) and 3,5'-dimethyl-5,3'-di-tert-butyl diphenoquinone (35DPQ) dispersed in polystyrene (PS) or poly $\left(4,4^{\prime}\right.$-cyclohexylidenediphenyl)polycarbonate $(\mathrm{PC}-\mathrm{Z}) .{ }^{[47]}$

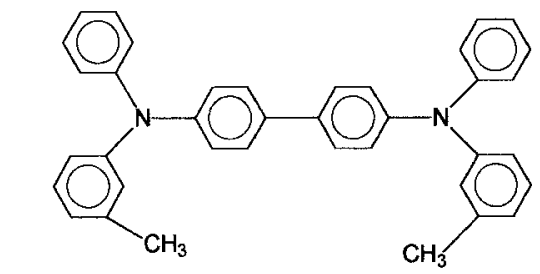

TPD<smiles>CCN(CC)c1ccc(C(C)NN(c2ccccc2)c2ccccc2)cc1</smiles>

Sometimes the initial spike of the transient photocurrents is followed by a cusp rather than by a plateau. This was attributed to the occurrence of space charges or trapping of charges at the interface. ${ }^{[24.29]}$ More recently, Borsenberger et al. ${ }^{[30]}$ have also reported a similar phenomenon in transient photocurrents obtained using an $\alpha$-Se layer as a photoemitting electrode, but in this case it was attributed to energy-selective carrier injection at the interface between the emitting electrode and the transport layer.

\section{Field and Temperature Dependence of the Mobilities}

As only a very small fraction of the sites have an energy equal to or smaller than $\left\langle\varepsilon_{\infty}\right\rangle=-\sigma^{2} /(k T)$, hopping will require an activation with a barrier of the same order of magnitude as $\left\langle\varepsilon_{\infty}\right\rangle$. Therefore one can expect that the hopping is activated and that the activation energy is proportional to $1 / T$. This will yield a non-Arrhenius temperature dependence of the mobility. On the other hand, applying an electric field $E$ will tilt the DOS and thus reduce the activation energy for uphill jumps in the field direction. The Monte Carlo calculations suggest that $\left\langle\varepsilon_{\infty}\right\rangle$ varies according to Equation 19. ${ }^{[36,37]}$

$\left\langle\delta_{\infty}\right\rangle=-\sigma^{2} /(k T)+\left(E / E_{0}\right)^{3 / 2}$

In Equation 19, $E_{0}$ does not depend upon $\sigma$ and equals $1.8 \times 10^{6} \mathrm{Vcm}^{-1}$ for an average intermolecular distance of
$6 \AA$. In the framework of the hopping in a Gaussian DOS with superimposed positional disorder the following dependence of the charge carrier mobility upon the applied field and the temperature was obtained for field strengths ranging between $1.0 \times 10^{5}$ and $1.0 \times 10^{6} \mathrm{Vcm}^{-1}$. Here $\mu_{00}$ is the mo-

$$
\begin{array}{r}
\mu=\mu_{00} \exp \left[-\left(\frac{2 \sigma}{3 k T}\right)^{2}\right] \exp \left\{E^{1 / 2} C\left[\left(\frac{\sigma}{k T}\right)^{2}-\Sigma^{2}\right]\right\} \\
\text { for } \Sigma \geq 1.5 \\
\mu=\mu_{00} \exp \left[-\left(\frac{2 \sigma}{3 k T}\right)^{2}\right] \exp \left\{E^{1 / 2} C\left[\left(\frac{\sigma}{k T}\right)^{2}-2.25\right]\right\} \\
\text { for } \Sigma<1.5
\end{array}
$$

bility at infinite absolute temperature and at zero field, $T$ is the absolute temperature $[\mathrm{K}], \sigma$ is the width of the DOS [eV], $k$ is the Boltzmann constant $\left(1.38 \times 10^{-23} \mathrm{JK}^{-1}\right), \Sigma$ is a parameter describing the off-diagonal disorder, and $C$ is an empirical constant. $C$ is expected to scale with the square root of the intermolecular distance and equals $2.9 \times 10^{-4}\left(\mathrm{~cm} \mathrm{~V}^{-1}\right)^{1 / 2}$ for an average intermolecular distance of $6 \AA$. The field and temperature dependence of the mobility $\mu$, reported in Equation 20, were obtained using Monte Carlo calculations ${ }^{[36,37]}$ to describe the average position and energy of a charge carrier in a Gaussian density of states in the presence of an applied electric field. The hopping rate constant between two neighboring sites was assumed to be given by Equation 16 . Using an effective medium theory ${ }^{[28]}$ an analogous dependence was obtained.

The key predictions of Equation 20 are that the logarithm of the mobility is proportional to the square root of the applied field and to the inverse temperature squared. A further prediction is that the slope of the field dependence of the mobility $(\partial \ln \mu / \partial \sqrt{E})$ is proportional to $T^{-2}$. Simulations for other types of DOS always suggest a linear dependence between $\ln \mu$ and $E^{n}$ with $0 \leq n \leq 0.5 \cdot{ }^{[52]}$ Equation 20 describes adequately the field and temperature dependence of the mobility obtained for a large number of hole transport materials dispersed in a polymer matrix or as vapor deposited layers. Plotting $\ln \mu$ versus $E^{1 / 2}$ yields a linear relationship with a slope equal to $C\left[(\mathrm{e} \sigma /(k T))^{2}-2.25\right]$ or $C\left[(e \sigma /(k T))^{2}-\Sigma^{2}\right]$ and an intercept equal to $\ln \left(\mu_{00}\right)-(2 \sigma e /(3 k T))^{2}$. Plotting the logarithm of this intercept versus $1 / T^{2}$ yields again a linear relationship with a slope equal to $(2 \sigma / 3 k)^{2}$ and an intercept equal to $\ln \mu_{00}$.

In analogy to the Poole-Frenkel formalism, $\ln \mu$ is proportional to $E^{1 / 2}$. However, while, beside other objections, it was difficult to attribute a physical interpretation to the large density of charged traps obtained by application of the Poole-Frenkel mechanism, this is no longer a problem for the hopping in a Gaussian DOS. A more recent interpretation, suggesting that the agreement with a Poole-Frenkel relationship is due to the presence of dipolar traps, cannot, however, be excluded. ${ }^{[53]}$ As observed for several systems, the slope of $\ln \mu$ versus $E^{1 / 2}$ (Fig. 6) decreases with increasing temperature and can even 


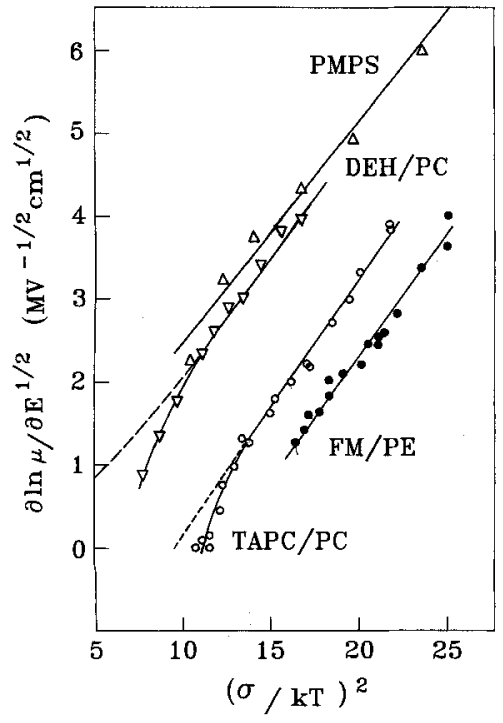

Fig. 6. The slope $S$ of $\ln \mu$ versus $E^{1 / 2}$ plots [36] for various systems: $\Delta$, PMPS $[57,62] ; \nabla, \mathrm{DEH} /$ polycarbonate $[49,50] ;$ o, TAPC/polycarbonate [39]; $\bullet$ FM $[88,89]$ in polyester.

become negative when e $\sigma /(k T)$ becomes smaller than $\Sigma$ (Fig. 7). This means that when the temperature is increased the mobility can become field independent or even decrease when the applied field is increased. Although the presence of off-diagonal disorder $(\Sigma>0)$ will lead to a sign reversal of the plots of $\ln \mu$ versus $E^{1 / 2}$ at lower temperatures, this phe-

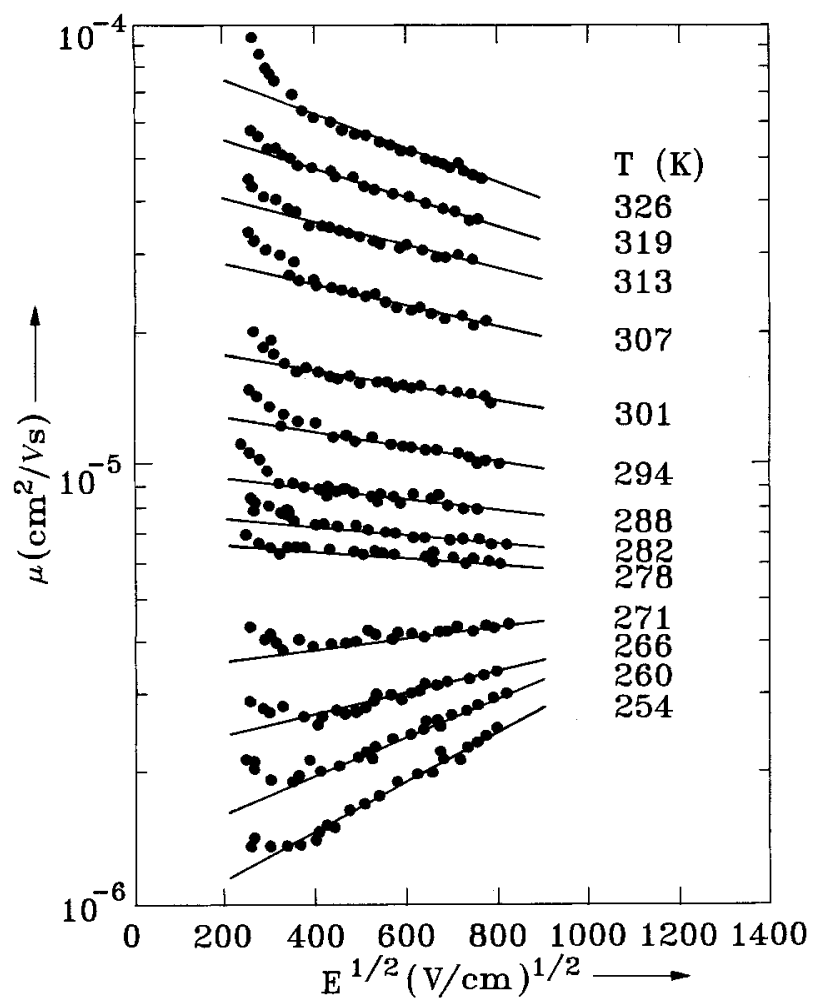

Fig. 7. Hole mobility versus $E^{1 / 2}$ in $75 \%$ TAPC in polycarbonate doped with $2 \% p$-dinitrobenzene, parametric in temperature $[39,40,80]$. nomenon should, contrary to earlier statements, ${ }^{[54]}$ also occur in the absence of off-diagonal disorder. According to Equation 20 the apparent activation energy of the mobilities extrapolated to zero field can be expressed as Equation 21.

$\lim _{E \rightarrow 0} E_{\mathrm{a}}(E)=\frac{8 \sigma^{2}}{9 k T}$

When the mobilities were determined over a large temperature range the analysis of the temperature dependence of the mobilities extrapolated to zero field always yielded a better fit in the framework of Equation 20 than in the framework of an Arrhenius expression. Furthermore, while analysis of the mobilities extrapolated to zero field strength using an Arrhenius expression yielded values of the mobility at zero field strength and infinite temperature that were two to three orders of magnitude larger than those obtained for single crystals, ${ }^{[55,56]}$ values of the same order of magnitude or smaller than those obtained for single crystals were found when Equation 20 was used. Upon increasing the applied field the apparent activation energy decreases according to Equation 22.

$E_{\mathrm{a}}(E)=\left(\frac{4}{9}-C E^{1 / 2}\right) \frac{2 \sigma^{2}}{k T}$

Equation 22 describes the field dependence of the activation energy observed for a large number of systems, ranging from conjugated $^{[57-60]}$ and non-conjugated polymers ${ }^{[61,62]}$ to molecularly dispersed hole transport materials. ${ }^{[42]}$ The correspondence ${ }^{[63]}$ between the values of the zero field activation energies and mobilities obtained by extrapolation of Equation 20 or 22 and the values obtained by the determination of the hole diffusion coefficient in the absence of an applied field supports the use of Equations 20 and 22. Simulations also suggest that at low fields $\ln \mu$ saturates upon a further decrease of field. This effect occurs apparently at higher field strengths when $\sigma$ increases. This effect was experimentally observed for poly(methylphenylsilane), PMPS. ${ }^{[58,63]}$ Saturation occurred at lower field strengths than suggested by the simulations. This was due to the different field dependence of the values of $t_{0}$ and the values of $t_{t r}$ obtained at low fields on the basis of Equation $6^{[64]}$ This discrepancy is due to the field dependence of the features of the non-dispersive transient photocurrents at low applied fields. One has furthermore to take into account that at low applied fields the features of the transient photocurrents can be influenced by capture of the holes by a low concentration of deep traps or by slow geminate dissociation in the bulk.

According to simulations in the presence of off-diagonal disorder, the saturation observed at low field strengths in the plots of $\ln \mu$ versus $E^{1 / 2}$ is replaced by an increase of the mobility upon decreasing the field strength (Fig. 8). This phenomenon, which occurs at field strengths smaller than $1.0 \times 10^{6} \mathrm{Vcm}^{-1}$ is accompanied by a decrease of the slope of the plot of $\ln \mu$ versus $E^{1 / 2}$ at higher field strengths when Equation 20 is 


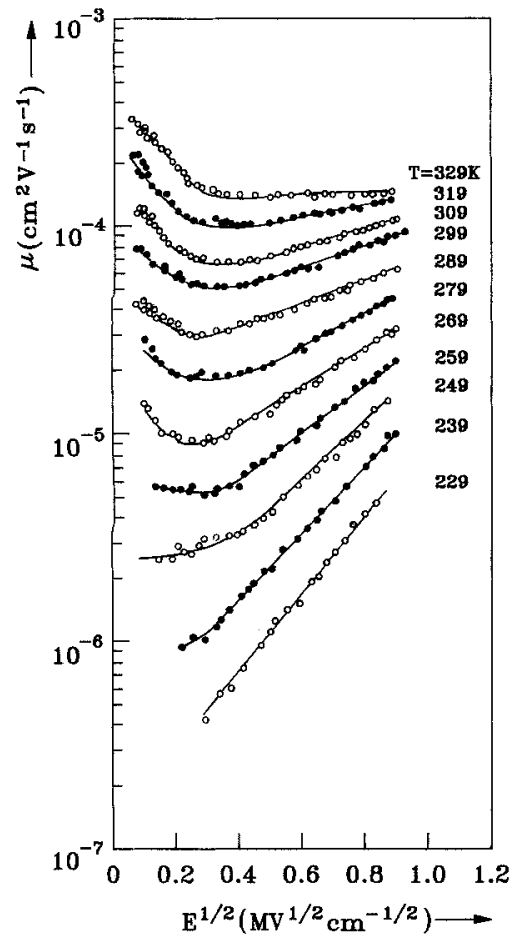

Fig. 8. Hole mobility versus $E^{1 / 2}$ in TAPC in polycarbonate, parametric in temperature [39].

valid. It is due to the fluctuations of the wavefunction overlap parameter $\Gamma_{i}$ yielding an asymmetric variation of the overlap given by $\exp \left(-\Gamma_{i}\right)$, Equation 16 . Due to these fluctuations, some indirect paths characterized by a favorable overlap but containing steps against the direction of the applied field can be faster at low fields than the direct path containing only steps in the direction of the applied field. In order to take full advantage of this effect, a carrier has to diffuse isotropically. Hence in zero field the carriers arrive earlier at an acceptor site by routes involving backward jumps than by executing only nearest neighbor jumps governed by an overlap factor $\exp (-\gamma a)$. As the applied field is increased, backward jumps will be impeded and the "indirect routes" will become inhibited, inducing a decrease of the mobility. At larger fields this effect will saturate and become compensated by the effects of the tilting of the DOS caused by the applied field. When the wavefunction overlap parameter is characterized by a Gaussian distribution, Equation 23, the average diffusion coefficient corresponding to $\left\langle\exp \left[-2 g\left(\Gamma_{i}\right)\right]\right\rangle$ will be given by Equation 24 . Here $D(\Sigma=0)$ corresponds

$$
\begin{aligned}
& \mathrm{g}\left(\Gamma_{i}\right)=\frac{1}{\pi \Sigma} \exp \left[-\frac{\left(\Gamma_{i}-\gamma a\right)^{2}}{\Sigma^{2}}\right] \\
& \langle D(\Sigma)\rangle=D(\Sigma=0) \exp \left(\Sigma^{2} / 2\right)
\end{aligned}
$$

to the diffusion coefficient in the absence of off-diagonal disorder. Hence off-diagonal disorder leads to an increase of the mobility by a factor $\exp \left(\Sigma^{2} / 2\right)$. The effect of off-diagonal disorder on the field dependence of the hole mobility could be observed very clearly between $279 \mathrm{~K}$ and $329 \mathrm{~K}$ for a molecular dispersion of TAPC in polycarbonate. ${ }^{[39]}$ The saturation of the mobility at low fields has also been observed for polyvinylcarbazole. ${ }^{[65]}$ At very large applied fields where only steps in the direction of the field are allowed (eaE $\gg k T$ ) and where the tilting of the DOS is so strong that all forward steps are exothermic $v_{i j}$ will no longer depend upon the applied field. In this case $v_{\mathrm{dr}}$ will saturate and hence the mobility will become proportional to the inverse field $\left(\mu \propto E^{-1}\right)$. Under these conditions one would expect, in the framework of the disorder model, that the decrease of the activation barrier will make the mobility nearly independent of the temperature.

As shown in Table 1, increasing the concentration of a dispersion of TTA in polycarbonate leads to an increase of $\mu_{00}$ and a decrease of $\sigma$. This is accompanied by a less important decrease

Table 1. Influence of the concentration on the hole transport parameters of TTA in polycarbonate.

\begin{tabular}{llllll}
\hline $\begin{array}{l}\text { Conc. } \\
\text { of TTA }\end{array}$ & $\mu_{00}$ & $\sigma$ & $\Sigma$ & $C$ & $\mu_{00} \exp \left(-\Sigma^{2} / 2\right)$ \\
{$\left[\mathrm{wt}{ }^{-\%}\right]$} & {$\left[\mathrm{cm}^{2} \mathrm{~V}^{-1} \mathrm{~s}^{-1}\right]$} & {$[\mathrm{eV}]$} & & {$\left[\left(\mathrm{cm} \mathrm{V}^{-1}\right)^{1 / 2}\right]$} & {$\left[\mathrm{cm}^{2} \mathrm{~V}^{-1} \mathrm{~s}^{-1}\right]$} \\
\hline 10 & $6.0 \times 10^{-4}$ & 0.150 & 4.36 & $2.68 \times 10^{-4}$ & $4.5 \times 10^{-8}$ \\
14 & $1.0 \times 10^{-3}$ & 0.135 & 3.92 & $3.11 \times 10^{-4}$ & $4.6 \times 10^{-7}$ \\
20 & $4.0 \times 10^{-3}$ & 0.123 & 4.50 & $2.21 \times 10^{-4}$ & $1.6 \times 10^{-7}$ \\
30 & $5.5 \times 10^{-2}$ & 0.116 & 4.50 & $2.06 \times 10^{-4}$ & $2.2 \times 10^{-6}$ \\
40 & $1.0 \times 10^{-1}$ & 0.116 & 3.71 & $2.62 \times 10^{-4}$ & $1.0 \times 10^{-4}$ \\
50 & $1.5 \times 10^{-1}$ & 0.108 & 3.60 & $2.79 \times 10^{-4}$ & $2.3 \times 10^{-4}$ \\
\hline
\end{tabular}

of $\Sigma^{[44]}$ The variation of $\sigma$ as a function of the concentration will be discussed in the following section. In the framework of Equation 16 the increase of $\mu_{00}$ is due to a decrease of $\gamma a$. A plot of $\ln \mu_{00}$ deviates at low and high concentration from the linear relationship expected on the basis of Equation 16. The deviations at high concentrations may be due to the onset of percolation. ${ }^{[66]}$ However, if this effect is taken into account the experimental values of $\mu_{00}$ at low concentration are still larger than those calculated on the basis of the model of Ries. This can be due to an increase of $\Sigma$ at low concentration leading to an increased enhancement of $\mu_{00}$. To separate the effects of the concentration on the enhancement of the mobility by off-diagonal disorder or by an increase of the average overlap, $\mu_{00}$ has to be multiplied by $\exp \left(-\Sigma^{2} / 2\right)$.

\section{Influence of Molecular Properties on the Transport Parameters}

For polymers containing functions characterized by a permanent dipole moment (carbonyl, ester, sulfone, halogens) or a large polarizability, variations of the mutual orientation or distance between the polymer host and the transport molecule will cause variations of the solvation energy of the charge carrier. One expects the distribution of solvation energies to become wider when the polarizability of the polymer or the dipole moments of the polar functions in the polymer become 
larger. ${ }^{[34,35]}$ This will lead to larger value of $\sigma$ and a lower mobility. It has been reported that for trans-1,2-bis $(9 H$-carbazole)cyclobutane (DCZB), ${ }^{[67]} N, N^{\prime}$-diphenyl- $N, N^{\prime}$-bis(3methylphenyl)-(1,1-biphenyl)-4, $4^{\prime}$-diamine (TMPD) ${ }^{[68]}$ or 2-( $p$-dipropylaminophenyl)-4-( $p$-dimethylaminophenyl)-5(o-chlorophenyl)-1,3-oxadiazole ${ }^{[69]}$ the hole mobility is decreased upon increasing the permanent dipole moments of groups in the main or side chains of the host polymers. A similar result was observed upon increasing the dipole moment of hole transport molecules dispersed in polyvinylbutyral. ${ }^{[70]}$ Also the influence of the substituents on the hole mobility of triarylmethanes can be attributed to a change of the molecular dipole moment. ${ }^{[51,71]}$ For a series of hydrazones it was suggested that increasing the delocalization of the positive charge leads to a reduction of $\sigma$ and an increase of $\mu_{00}{ }^{[72]}$ According to Vannikov similar results observed upon addition of polar molecules to a polymer doped with triphenylamine ${ }^{[73]}$ were attributed to an increase of the polaron binding energy. ${ }^{[74-78]}$ On the other hand the addition of the electron transport compound 3,5-dimethyl-3',5'-di-tert-butyl-diphenoquinone (55DPQ) to a molecular dispersion of $N, N . N^{\prime}, N^{\prime}$-tetra( $m$ tolyl)-1,3-diaminobenzene (PDA) ${ }^{[79]}$ did not increase the zero field activation energy but decreased the zero field mobility extrapolated to infinite temperature by two orders of magnitude.

The transport parameters $\left(\mu_{00}, \sigma\right.$, and $\left.C \Sigma^{2}\right)$ obtained from the analysis of the field and temperature dependence of the hole mobility of TTA dispersed in several polymers revealed ${ }^{[43]}$ that while the different polymers were characterized by similar values of $\Sigma^{2}$ they showed large variations of $\sigma$ and $\mu_{00}$. To some extent the apparent increase in $\sigma$ seems to be accompanied by a decrease of $\mu_{00}$. Although the changes of $\sigma$ seem small, Table $2 \mathrm{a}$ shows that at $298 \mathrm{~K}$ the variation of $\exp [-(2 e \sigma /$ $\left.(3 k T))^{2}\right]$ is as least as important as that of $\mu_{00}$. This would suggest that the polarity of the polymers increases from polycarbonate over the polyesters to the polysulfone. The larger value of $\sigma$ obtained for polymers with the ester group in the side chain compared to polyester or polycarbonate (Table $2 \mathrm{a}$ and Table 2 c) can be attributed to a larger accessibility of this side group to the transport molecule. The variations in $\mu_{00}$ can be related to variations in the wavefunction overlap parameter $\gamma$ and variations in the off-diagonal disorder.

For polystyrene, ${ }^{[80]}$ which contains no functions with a permanent dipole moment, an important decrease of $\sigma$ accompanied by an increase of $\mu_{00}$ is observed (Table $2 \mathrm{~b}$ ). In polystyrene the variations in the stabilization of the hole will mainly be due to fluctuations of the polarization energy caused by local variations of the intermolecular distances and orientations. ${ }^{[34]}$ Another intramolecular contribution to $\sigma$ may arise from a distribution of intramolecular rotation angles of, for example, the phenyl groups in the transport molecule. ${ }^{[81]}$ In practice, if the different contributions to the width of the DOS can be considered independent, the total width of the DOS is given by Equation 25 .

$\sigma=\left(\sum_{j} \sigma_{j}^{2}\right)^{1 / 2}$
Table 2. Influence of the matrix on the transport properties of hole transporting materials.

a) For a dispersion of $40 \mathrm{wt} \%$ TTA. $\mu_{00}\left[\mathrm{~cm}^{2} \mathrm{~V}^{-1} \mathrm{~s}^{-1}\right] \sigma[\mathrm{eV}] \quad \Sigma \quad C\left[\left(\mathrm{cmV}^{-1}\right)^{1 / 2}\right] \exp [-2 e \sigma /$ $\left.(3 k T)^{2}\right][\mathrm{a}]$

\begin{tabular}{|c|c|c|c|c|c|}
\hline Polycarbonate & 0.01 & 0.123 & 3.77 & $2.60 \times 10^{-4}$ & $2.15 \times 10^{-5}$ \\
\hline Polyester & 0.034 & 0.129 & 3.52 & $2.65 \times 10^{-4}$ & $7.5 \times 10^{-6}$ \\
\hline $\begin{array}{l}\text { Poly(methyl } \\
\text { methacrylate) }\end{array}$ & 0.0061 & 0.132 & 3.53 & $2.57 \times 10^{-4}$ & $4.15 \times 10^{-6}$ \\
\hline $\begin{array}{l}\text { Poly(vinyl-3- } \\
\text { bromobenzoate) }\end{array}$ & 0.0069 & 0.137 & 3.75 & $2.55 \times 10^{-4}$ & $1.65 \times 10^{-6}$ \\
\hline Polysulfone & 0.0059 & 0.149 & 3.22 & $2.78 \times 10^{-4}$ & $1.27 \times 10^{-7}$ \\
\hline
\end{tabular}

b) For a dispersion of TAPC.

\begin{tabular}{|c|c|c|c|c|}
\hline & $\mu_{00}\left[\mathrm{~cm}^{2} \mathrm{~V}^{-1} \mathrm{~s}^{-1}\right]$ & $\sigma[\mathrm{eV}]$ & $\Sigma$ & $C\left[\left(\mathrm{~cm} \mathrm{~V}^{-1}\right)^{1 / 2}\right]$ \\
\hline Evaporated layer & 0.13 & 0.068 & 1.00 & $2.5 \times 10^{-4}$ \\
\hline Polystyrene [b] & 0.23 & 0.077 & 2.14 & $2.54 \times 10^{-4}$ \\
\hline Polycarbonate $[\mathrm{b}]$ & 0.04 & 0.095 & 3.1 & $3.01 \times 10^{-4}$ \\
\hline $\begin{array}{l}\text { Polycarbonate } \\
+o \text {-DNB [c] }\end{array}$ & 0.01 & 0.118 & 4.4 & $2.82 \times 10^{-4}$ \\
\hline $\begin{array}{l}\text { Polycarbonate } \\
\quad+m \text {-DNB [d] }\end{array}$ & 0.14 & 0.119 & 4.7 & $2.84 \times 10^{-4}$ \\
\hline $\begin{array}{l}\text { Polycarbonate } \\
\quad+p \text {-DNB [e] }\end{array}$ & 0.21 & 0.115 & 5.0 & $3.07 \times 10^{-4}$ \\
\hline
\end{tabular}

c) For a dispersion of 40 wt $-\%$ of DCZB.

\begin{tabular}{llll}
\hline & $\mu_{00}\left[\mathrm{~cm}^{2} \mathrm{~V}^{-1} \mathrm{~s}^{-1}\right] \sigma[\mathrm{eV}]$ & $\exp \left[-2 e \sigma /(3 k T)^{2}\right][\mathrm{a}]$ \\
\hline $\begin{array}{c}\text { Polycarbonate } \\
\begin{array}{c}\text { Poly(hexyl } \\
\text { methacrylate) }\end{array}\end{array}$ & 0.0011 & 0.138 & $1.315 \times 10^{-6}$ \\
$\begin{array}{c}\text { Poly(isobutyl } \\
\text { methacrylate) }\end{array}$ & 0.0063 & 0.138 & $1.31 \times 10^{-6}$ \\
$\begin{array}{c}\text { Polystyrene } \\
\begin{array}{c}\text { Poly(benzyl } \\
\text { methacrylate) }\end{array}\end{array}$ & 0.0079 & 0.140 & $8.85 \times 10^{-7}$ \\
$\begin{array}{c}\text { Poly(propyl } \\
\text { methacrylate) }\end{array}$ & 0.0020 & 0.144 & $3.95 \times 10^{-7}$ \\
$\begin{array}{c}\text { Poly(methyl } \\
\text { methacrylate) }\end{array}$ & 0.0063 & 0.152 & $7.32 \times 10^{-8}$ \\
\hline
\end{tabular}

[a] At $298 \mathrm{~K}$. [b] Concentration $75 \mathrm{wt}-\%$, [c] Concentration $75 \mathrm{wt}-\%+2 \%$-dinitrobenzene. [d] Concentration $75 \mathrm{wt} \%+2 \% \mathrm{~m}$-dinitrobenzene. [e] Concentration $75 w \mathrm{t}-\%+2 \% p$-dinitrobenzene.

As reported in Table $2 \mathrm{~b}$, the dispersion of molecules with a large dipole moment in the polymer matrix will give rise to additional contributions to $\sigma$. This does not imply that there exists a simple relation between the dipole moment of the matrix or polar additives and $\sigma$. Although $o$-DNB ( $o$-dinitrobenzene), $m$-DNB ( $m$-dinitrobenzene), and $p$-DNB ( $p$-dinitrobenzene) are characterized by permanent dipole moments of $6 \mathrm{D}, 4 \mathrm{D}$, and $0.5 \mathrm{D}$, respectively, a similar increase of $\sigma$ is observed. ${ }^{[40,82]} \mathrm{A}$ possible explanation could be that, as dipole-ion interactions are characterized by a strong distance dependence $\left(1 / r^{2}\right)$, the hole interacts only with the closest nitro group. It is also possible that the additives work in an indirect way by changing the packing of the polymer of the transport molecule. The latter argument is supported by the changes of $\Sigma$ induced by the additives. Although for $\operatorname{DCZB}^{[67]} \sigma$ increases between polycarbonate and polymethylmethacrylate, this is not accompanied by an increase between polystyrene and polycarbonate. This dis- 
crepancy could be explained by, for example, clustering or formation of microcrystals by DCZB in polystyrene (Table 2c).

For vapor-deposited layers, the data reported in Table 3 suggest that $\sigma$ increases when the dipole moment of the transport molecule is increased. ${ }^{[83]}$ The large value of $\sigma$ observed for MPMP is due to the fact that MPMP contains two diethylamino groups with almost opposite dipole moments. On the other hand, the random electric fields contributing to energetic disorder must be local fields in the immediate vicinity of a polar group. In polar polymers (polycarbonate or polyester) this effect is much smaller. This is due to the more important interactions between the dipole moments of dipolar moieties of the polymer and the hole compared to the interaction between the hole and the (small) dipole moments of the transport materials. For electron transport materials dispersed in polymers (Table $3 \mathrm{c}$ ) the width of the DOS is always much larger than that of hole transporting molecules. This can be explained by the large dipole moments of the cyano or cyanovinylidene groups. Furthermore, the size of the latter molecules is always considerably smaller. As the energy of ion-dipole interactions (of the polymer or of transport molecules) is proportional to

Table 3. Influence of the chemical structure on the transport properties.

a) Amorphous evaporated layers of hole transport materials or polymeric transport materials.

\begin{tabular}{llllll}
\hline & $\mu_{\mathbf{0} 0}\left[\mathrm{~cm}^{2} \mathrm{~V}^{-1} \mathrm{~s}^{-1}\right]$ & $\sigma[\mathrm{eV}]$ & $\Sigma$ & $C\left[(\mathrm{~cm} \mathrm{~V}-1)^{1 / 2}\right]$ & $\boldsymbol{\mu}[\mathrm{D}]$ \\
\hline TAPC & 0.13 & 0.067 & 1.00 & $2.5 \times 10^{-4}$ & 1.00 \\
MPMP [a] & 0.34 & 0.098 & 2.0 & {$[\mathrm{~b}]$} & 1.23 \\
TPD & 0.035 & 0.074 & 1.2 & $3.6 \times 10^{-4}$ & 1.52 \\
TTB [c] & 0.019 & 0.069 & 1.5 & {$[\mathrm{~b}]$} & 1.56 \\
pEFTP & 0.069 & 0.068 & 1.0 & $3.0 \times 10^{-4}$ & 1.90 \\
DEH & 0.0013 & 0.100 & 2.0 & $3.2 \times 10^{-4}$ & 3.17 \\
DEASP [d] & 0.0060 & 0.103 & 1.4 & {$[\mathrm{~b}]$} & 4.34 \\
PMPS [e] & 0.015 & 0.094 & 0.0 & $2.64 \times 10^{-4}$ & {$[\mathrm{~b}]$} \\
\hline
\end{tabular}

b) Dispersion of hole transport materials in polycarbonate.

\begin{tabular}{llllll}
\hline & $\mu_{00}\left[\mathrm{~cm}^{2} \mathrm{~V}^{-1} \mathrm{~s}^{-1}\right]$ & $\sigma[\mathrm{eV}]$ & $\Sigma$ & $C\left[\left(\mathrm{~cm} \mathrm{~V}^{-1}\right)^{1 / 2}\right]$ & $\mu[\mathrm{D}]$ \\
\hline TAPC [f] & 0.040 & 0.095 & 3.1 & $3.01 \times 10^{-4}$ & 1.00 \\
pEFTP [g] & 0.051 & 0.124 & 4.1 & $2.12 \times 10^{-4}$ & 1.9 \\
TTA [f] & 0.010 & 0.120 & 3.77 & $2.60 \times 10^{-4}[\mathrm{~h}]$ & {$[\mathrm{b}]$} \\
DCZB & 0.0011 & 0.138 & {$[\mathrm{~b}]$} & {$[\mathrm{b}]$} & {$[\mathrm{b}]$} \\
DEH [i] & 0.00093 & 0.122 & 3.90 & $3.46 \times 10^{-4}$ & \\
\hline
\end{tabular}

c) Dispersion of electron transport materials in polymere

\begin{tabular}{lllll}
\hline & $\mu_{00}\left[\mathrm{~cm}^{2} \mathrm{~V}^{-1} \mathrm{~s}^{-1}\right]$ & $\sigma[\mathrm{eV}]$ & $\Sigma$ & $C\left[\left(\mathrm{~cm} \mathrm{~V}^{-1}\right)^{1 / 2}\right]$ \\
\hline DPQ [j] & 0.0023 & 0.118 & 3.30 & $2.90 \times 10^{-4}$ \\
DPQ [k] & 0.035 & 0.156 & 2.90 & $2.90 \times 10^{-4}$ \\
FM [1,m] & 0.0033 & 0.138 & 3.6 & $2.90 \times 10^{-4}$
\end{tabular}

[a] (MPMP)

[b] No data available. [c] $N, N, N^{\prime}, N^{\prime}$-Tetrakis(4-methylphenyl)(1,1'-biphenyl)4,4'-diamine (TTB). [d] 1-Phenyl-3[p-(diethylamino)styryl]-5-[ $p$-(diethylamino)phenyllpyrazoline (DEASP) [84]. [e] From analysis of Poole-Frenkel plots in $[58,63]$. [f] $40 \mathrm{wt}-\%$. [g] $30 \mathrm{wt}-\%$. [h] $C$ is obtained from [36] with $\beta=\sigma^{2} C$, $\left(k^{2} T\right)$. [i] $50 \mathrm{wt}-\%$. [j] Polystyrene. [k] Poly $\left(4,4^{\prime}\right.$-cyclohexylidenediphenyl)carbonate. [l] 4-(n-Butoxycarbonyl)-9-fluorenylidenemalononitrile. [m] Polyester. the inverse square of the distance, similar dipole moments will give rise to a larger solvation energy and to a wider distribution of solvation energies for electron transporting materials. Furthermore, one should take into account that DPQ is a mixture of two isomers, which could lead to an additional broadening of the DOS. Although $\Sigma$ is smaller for vapor-deposited materials than for molecular dispersions, it is not possible to observe a systematic relation between $\Sigma$ and the chemical structure of the transport molecule.

To compare the different values obtained for $\mu_{00}$ it is necessary to multiply them by $\exp \left(-\Sigma^{2} / 2\right)$ to correct for differences in off-diagonal disorder. With the exception of TAPC, values ranging between $1.7 \times 10^{-5}$ and $3.3 \times 10^{-6} \mathrm{~cm}^{2} \mathrm{~V}^{-1} \mathrm{~s}^{-1}$ are obtained for molecular dispersions containing 40 or $45 \mathrm{wt}-\%$ of the transport material. Only for TAPC dispersed in polycarbonate does $\mu_{00} \exp \left(-\Sigma^{2} / 2\right)$ amount to $3.3 \times 10^{-4} \mathrm{~cm}^{2} \mathrm{~V}^{-1} \mathrm{~s}^{-1}$. The similarity of the parameters obtained for different compounds makes it unlikely that the features of the transient photocurrents are related to multiple trapping. ${ }^{[64,85]}$ It would be unlikely that chemically different systems would be characterized by a similar density of traps having the same depth relative to the transport level (isolated molecules or valence band).

\section{Field and Temperature Mobility of Dispersions of pEFTP in Polycarbonate}

The features of the transient photocurrents observed for hole transport by pEFTP dispersed in polycarbonate suggest the occurrence of hopping in a Gaussian DOS for concentrations between $11 \%$ and $60 \%$ and temperatures between $286 \mathrm{~K}$ and $347 \mathrm{~K}^{[42,45,46,86]}$ Therefore it was attempted to analyze the field and temperature dependence of the hole mobility data of a dispersion of $\mathrm{pEFTP}$ in polycarbonate for a concentration (weight fraction) ranging between $24 \%$ and $60 \%$ using Equation 20. Figure 9 shows that at $298 \mathrm{~K} \ln \mu$ is proportional to $E^{1 / 2}$ for concentrations ranging for 24 to $60 \mathrm{wt}-\%$. The

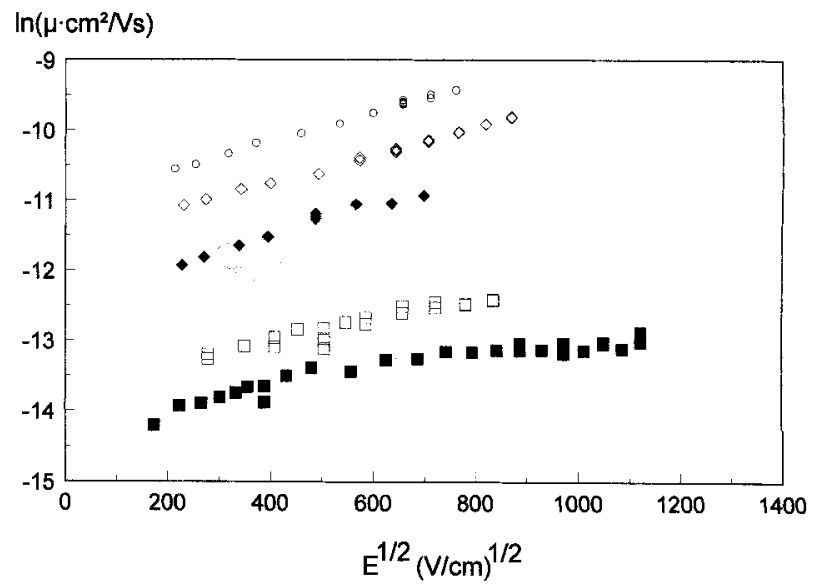

Fig. 9. Hole mobility in pEFTP in polycarbonate at $298 \mathrm{~K}$ versus $E^{1 / 2}$, pardmetric in concentration: a, $24 \mathrm{wt}-\%$; 口, $30 \mathrm{wt}-\% ; \bullet, 40 \mathrm{wt}-\% ; \diamond, 50 \mathrm{wt}-\%$; $\circ$, 60 wt- $\%$ [42]. 
intercepts, corresponding to $\mu(E=0)$, increase with increasing concentration. As the concentration is increased, the effective intermolecular distance becomes smaller, leading to an increase of $\exp (-\gamma a)$ in Equation 16. The slopes corresponding to $C\left[\sigma^{2} /(k T)^{2}-\Sigma^{2}\right]$ decrease gradually when the concentration is decreased, as observed by Yuh and Pai ${ }^{[68]}$ for $\left(N, N^{\prime}\right.$-diphenyl-bis-(3-methylphenyl)-[1,1'-biphenyl]-4.4'-diamine), (TPD), and $N, N^{\prime}$-bis-(4-methylphenyl)- $N, N^{\prime}$-bis-(4-ethylphenyl)-[1,1'$3,3^{\prime}$-dimethylbiphenyl]-4,4'-diamine (ETPD). ${ }^{[70]}$ This is rather surprising in view of relation 26 between the empirical constant $C$, relating the field dependence of the mobility to the temperature dependence, ${ }^{[37]}$ and the concentration $c$.

$C \propto a^{-1 / 2} \propto c^{-1 / 6}$

On the basis of Equation 26 one would expect $C$ to increase by $17 \%$ when the concentration of pEFTP decreases from 60 to $24 \%$. This does not agree with the experimental results. Hence the decrease of $C\left[\sigma^{2} /(k T)^{2}-\Sigma^{2}\right]$ should therefore be attributed to a decrease of $\sigma$ or an increase of $\Sigma$ upon decreasing the concentration.

When the hole mobility in samples containing $30 \mathrm{wt}-\%$ pEFTP in polycarbonate (PC) is plotted versus the square root of the applied field a linear relationship parametric in temperature is obtained and the slope decreases with increasing temperature (Fig. 10). Analysis of the data, obtained by fitting

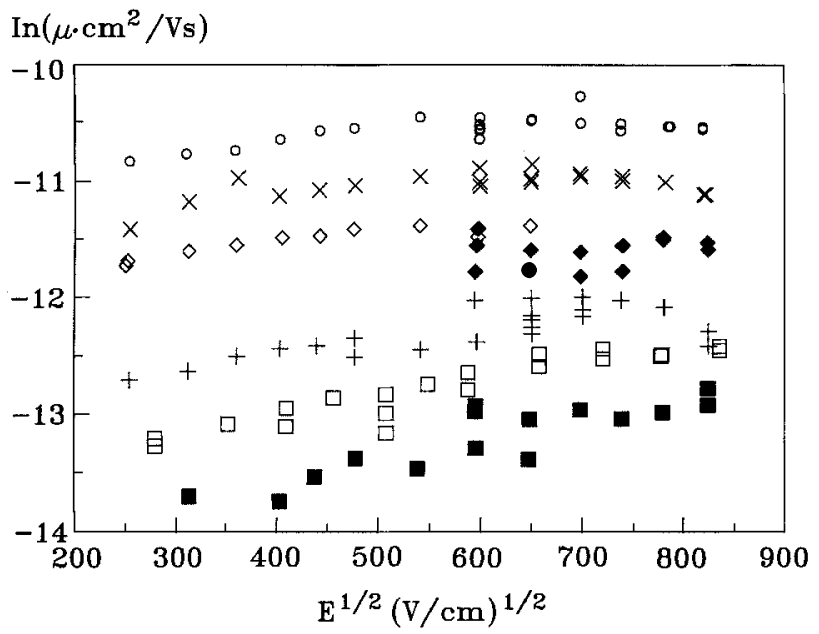

Fig. 10. Hole mobility in pEFTP $\left(30 \mathrm{wt} \%\right.$ ) in polycarbonate versus $E^{1 / 2}$, parametric in temperature: $\mathbf{m}, 286 \mathrm{~K} ; \square, 293 \mathrm{~K} ;+, 304 \mathrm{~K} ; \bullet, 318 \mathrm{~K} ; \diamond, 324 \mathrm{~K}$; $\times, 336 \mathrm{~K} ; 0,347 \mathrm{~K}[42]$

the experimentally measured transient photocurrents to Equation 17, in the framework of Equation 20 yields values for $\mu_{00}, T_{0}$ and $\sigma$ (Table 3 ) resembling those obtained for other similar molecules dispersed in polycarbonate. The values of $\Sigma$, although subject to a large experimental error, are similar to those that can be calculated for the data obtained by Borsenberger for a dispersion of TTA in polycarbonate. ${ }^{[4]}$ The values obtained for $\sigma$ resemble those calculated by the analysis of the mobilities obtained for a more dilute sample containing $17 \%$ pEFTP dispersed in polycarbonate. ${ }^{[46]}$ However, as was also observed for the analysis of the $17 \%$ sample, the values of $C$ are systematically smaller than predicted by simulations and the values found for other polyfunctional aromatic amines dispersed in polycarbonate. This discrepancy can be attributed to the largely anisotropic structure of pEFTP.

A closer inspection of the plots of $\ln \mu$ versus $E^{1 / 2}$ suggests that at high fields negative deviations occur from the proposed relation between $\ln \mu$ and $E^{1 / 2}$. This means that the deviations from the model developed for hopping in a Gaussian DOS that were observed for the more dilute samples ${ }^{[45,46]}$ are actually also present in this more concentrated sample. One could expect that this saturation or decrease of $\mu$ with increasing field is due to a saturation of the drift velocity. ${ }^{[37]}$ Considering that the free energy change associated with a jump of a charge carrier over a distance a equals $e E a$, the features of the plots of $\ln \mu$ versus $E^{1 / 2}$ obtained for different concentrations at a similar temperature should scale with $a$ or $c^{-1 / 3}$. The more important changes of the plots of $\ln \mu$ versus $E^{1 / 2}$ parametric in concentration, which were observed experimentally, can be attributed to changes of neither $\sigma$ nor $\Sigma$, as these parameters change only slightly when the concentration decreases from $30 \%$ (Table 3 ) to $17 \%{ }^{[46]}$ Furthermore, in this framework of the disorder model it is difficult to explain why the features of the plots of $\ln \mu$ versus $E^{1 / 2}$ change in such a drastic way when the temperature is changed. If the deviations from Equation 20 were due to saturation, the drift velocity would, in increasing field, asymptotically approach a limiting value that was independent of temperature. However, Figure 11 shows that for the sample containing $11 \%$ pEFTP the drift velocity, after reaching a maximum, starts to decrease upon a further increase of the applied field. As the

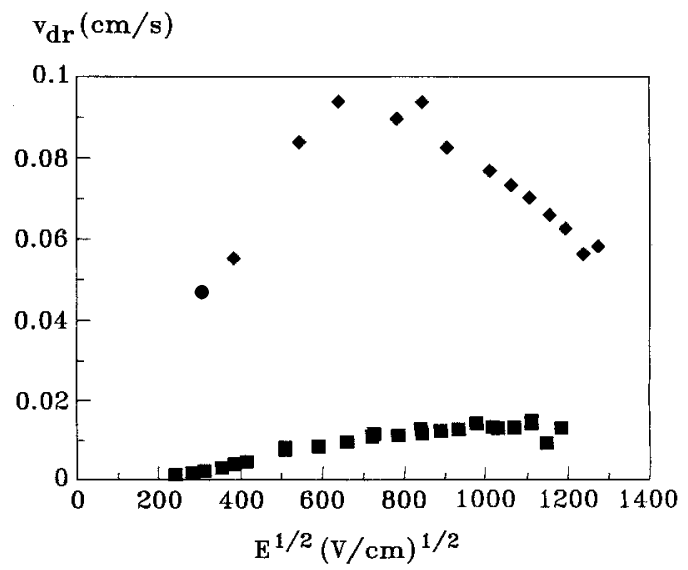

Fig. 11. Drift velocity in pEFTP (11\% weight) in polycarbonate versus $E^{1 / 2}$, parametric in temperature: $\mathbf{\bullet}, 298 \mathrm{~K}$;, $343 \mathrm{~K}[42,46]$.

anomalous field and temperature dependence is maximized under these conditions, similar effects can be expected for the other samples at higher applied fields or at higher temperatures. 
Such a decrease of the drift velocity, and hence of the jump rate, with increasing electric field corresponds to the dependence of the rate constants for electron transfer in the framework of Marcus's theory. ${ }^{[75-78]}$ This theory is actually another way of formulating the polaron model for charge transport. ${ }^{[87]}$ Therefore an alternative analysis in the framework of the polaron model was attempted for the data obtained for pEFTP.

\section{The Polaron Model}

The small polaron model ${ }^{[74,78]}$ was developed to describe charge transport in materials with small $(<0.05 \mathrm{eV})$ intermolecular interactions and hence narrow valence and conduction bands. In analogy with Marcus's theory ${ }^{[75-77]}$ this model is based on the instantaneous character of the electron transfer process compared to the rearrangement of the molecular geometry and the polarization of the environment. This polaron model is based on the change in the equilibrium values of bond lengths or bond angles or polarization of the environment when a neutral molecule is converted into the corresponding radical cation or radical anion. As this process is several orders of magnitude faster than the molecular vibrations, even processes which are thermoneutral will be characterized by an activation energy. When the electron transfer becomes exergonic an initial decrease of the activation barrier will be followed by an increase for strongly exergonic electron transfer processes. As the transport of charge carriers in amorphous materials can be considered as a sequence of uncorrelated electron transfer steps, there is no reason why the formalism developed for electron transfer processes should not be applied to charge carrier mobility. If those processes are important it will be necessary to take into account the changes of the equilibrium values of the bond lengths or the coupling to the medium upon oxidation or reduction when designing hole or electron transport materials.

When the free energy change associated with a hop is smaller than twice the polaron binding energy the effective forward or backward rate will be described by a hyperbolic sine (Eq. 27). In Equation 27, $\lambda$ corresponds to the reorganization energy and is related to the polaron binding energy by Equation 28.

$v_{\mathrm{dr}} \propto \exp \left(-\frac{\lambda}{4 k T}\right) \sinh \left(\frac{E e a}{2 k T}\right)$

$\lambda=2 E_{\mathrm{p}}$

Equation 27 would lead to an exponential increase of the drift velocity and hence of the mobility $\left(v_{\mathrm{dr}} / E\right)$. Gill[ ${ }^{[61]}$ was the first to describe charge transport in non-conjugated polymers by the polaron model. This model has been used later by several other authors. ${ }^{[87,90-93]}$ Although the temperature dependence of mobility data obtained for mixed transport materials can be analyzed using Equations 20 and 25 , the concentration dependence of the activation energy of the mobility in a mixed transport material can be explained in the framework of a polaron model. ${ }^{[94]}$

Except perhaps for a small range of fields close to zero this does not correspond to the experimental observations reported for pEFTP and other hole or electron transporting materials. On this basis the polaron model has been rejected for several systems. ${ }^{[37,81]}$ In the polaron model the effective rate constant of electron transfer between two sites is the sum of a forward and a backward reaction. ${ }^{[85]}$ The rate constants of both the forward $\left(k_{\mathrm{f}}\right)$ and the backward reaction $\left(k_{\mathrm{b}}\right)$ should be given by Marcus's theory. ${ }^{[77-79]}$

$k_{\mathrm{f}}=k_{0} \exp \left[-\left(\frac{\left(\Delta \mathrm{G}_{0}\right)^{2}}{4 k T \lambda}+\frac{\Delta \mathrm{G}_{0}}{2 k T}+\frac{\lambda}{4 k T}\right)\right]$

$k_{\mathrm{b}}=k_{0} \exp \left[-\left(\frac{\left(\Delta \mathrm{G}_{0}\right)^{2}}{4 k T \lambda}-\frac{\Delta \mathrm{G}_{0}}{2 k T}+\frac{\lambda}{4 k T}\right)\right]$

In these equations $\Delta G_{0}, k_{0}$, and $\lambda$ correspond to the free energy change associated with a forward hole or electron transfer, the value of $k_{\mathrm{f}}$ when $\Delta \mathrm{G}_{0}=-\lambda$, and the reorganization energy, respectively. $\Delta G_{0}=-e a E$ and $+e a E$ for a hole and an electron, respectively. Taking into account that the effective rate constant is the difference between the values obtained for the forward and backward processes, the mobility will be given by Equation 30 .

$\mu=\frac{a k_{0}}{E}\left[1-\exp \left(-\frac{E e a}{k T}\right)\right] \exp \left[-\frac{(-E e a+\lambda)^{2}}{4 \lambda k T}\right]$

In Equation $30 \Delta G_{0}$ has been replaced by - Eae and $+E a e$ for a hole and an electron, respectively. Equation 30 suggests that for $\lambda<0.1 \mathrm{eV}$ the mobility is independent of the applied fields at low fields. At high fields a decrease of the mobilities, which becomes more important when the hopping distance $a$ is increased, is expected. To evaluate the difference between Equations 27 and 30 one should realize that for a polaron binding energy of $0.1 \mathrm{eV} \Delta G_{0}$ already matches $\lambda$ at a field strength of $1.0 \times 10^{6} \mathrm{Vcm}^{-1}$ for a center-to-center distance of the transport molecules of $20 \AA$. For smaller polaron binding energies, the quadratic terms in the Marcus equation leading to the Marcus inverted region will become important even at lower field strengths. According to Sahyun, ${ }^{[95]}$ this second-order term is also necessary to describe the field dependence of hole mobility in polyvinylcarbazole. Contrary to Equation 27, Equation 30 no longer leads to an exponential dependence of the mobility on the applied field. Equation 27 or 30 yields a zero field mobility as in Equation 31 . When the matrix element $J$ coupling two neighboring sites is no longer negligible compared to $\lambda$, Equation 32 results.

$$
\begin{aligned}
& \mu_{0}=a^{2} e k_{0} \exp \left[-\frac{\lambda}{4 k T}\right] \\
& \mu_{0}=a^{2} e k_{0} \exp \left[-\frac{(\lambda / 4-J)}{k T}\right]
\end{aligned}
$$


In the non-adiabatic limit $k_{0}$ will be proportional to $J$ and hence depend upon the intermolecular distance $a$ and the concentration $c .^{[96]}$ Since $J$ becomes larger as the concentration of the transport molecules is increased, it is possible that $k_{0}$ will become independent of $J$ in the adiabatic limit. Under these conditions it is also possible that, as indicated in Equation 31 , the zero field activation energy decreases when $J$ is increased due to an increase of the concentration of the transport molecules. ${ }^{[96]}$ Although the analysis of the field dependence of the mobility in the framework of the polaron gives apparently consistent results for $\mathrm{DEH},{ }^{[96]} \mathrm{TTA}$, or $\mathrm{TPD},{ }^{[87,90-94]}$ it is difficult to compare the observed values for the zero field activation energy to values of the reorganization energy that are physically acceptable. Furthermore, this model yields values of $100 \mathrm{~cm}^{2} \mathrm{~V}^{-1} \mathrm{~s}^{-1}$ for $\mu_{00}$. It is difficult to understand why an amorphous system should give values for $\mu_{00}$ that exceed those of single crystals by two orders of magnitude.

The experimental data obtained for different concentrations of pEFTP at room temperature were analyzed in the framework of Equation 30 using $k_{0}, a$ and Table 4. Although for concentrations ranging from 17 to $50 \%$ realistic values were obtained for $\lambda$ and $k_{0}$ (Table 4), the values obtained for $a$ increase upon increasing the concentration of pEFTP. Also, at

Table 4. Analysis of the hole transport in pEFTP at $293 \mathrm{~K}$ in the framework of the hopping of a small polaron.

\begin{tabular}{lllll}
\hline $\begin{array}{l}\text { Conc. of pEFTP } \\
{[\mathrm{wt}-\%]}\end{array}$ & $k_{\mathrm{o}}\left[\mathrm{s}^{-1}\right]$ & $\lambda[\mathrm{eV}]$ & $a[\AA]$ & $\mu_{0 \mathrm{p}}\left[\mathrm{cm}^{2} \mathrm{~V}^{-1} \mathrm{~s}^{-1}\right][\mathrm{a}]$ \\
\hline 17 & $7.9 \times 10^{6}$ & 0.33 & 27.0 & $2.2 \times 10^{-5}$ \\
24 & $1.5 \times 10^{7}$ & 0.37 & 31 & $5.6 \times 10^{-5}$ \\
30 & $1.5 \times 10^{7}$ & 0.36 & 47 & $1.3 \times 10^{-4}$ \\
50 & $1.4 \times 10^{8}$ & 0.41 & 47 & $1.2 \times 10^{-3}$ \\
\hline
\end{tabular}

[a] $\mu_{\mathrm{op}}=a^{2} e k_{0} /(k T)$.

higher temperatures analysis of the data using Equation 30 does not yield combinations of $k_{0}, a$, and $\lambda$ that are physically acceptable. ${ }^{[46]}$ According to Equation 11, the free energy of activation of the mobilities extrapolated to zero field should amount to $\lambda / 4$. This is about five times less than the activation energies calculated using Equation 22 from the experimental values of $\sigma$. Furthermore, the values of $k_{0}$ would yield unrealistic values for $\mu_{00}$ exceeding those obtained for single crystals by several orders of magnitude.

For molecules such as pEFTP, the possible validity of the polaron model may also be discussed from a more speculative point of view. The polaron model relies on the relaxation of a transport molecule and its environment during the residence time of a charge carrier ( $\leq 100 \mathrm{~ns}$ ). Although apparently major conformational changes of the polymer are excluded in this time scale, the Stokes shift ${ }^{[97]}$ of a dilute ( $\left.0.1 \mathrm{wt}-\%\right)$ dispersion of pEFTP in polycarbonate suggests that some relaxation occurs during the lifetime of the excited singlet state ( $\leq 10 \mathrm{~ns}$ ). As the Stokes shift of the fluorescence of pEFTP in a dilute dispersion of polycarbonate resembles that in a solution of diethyl ether, one can expect that both systems are characterized by a similar reorganization energy. Assuming that electron-phonon coupling is limited to lowfrequency modes $(h v<k T)$ a value of $0.55 \mathrm{eV}$ can be obtained ${ }^{[98]}$ from the spectral data ${ }^{[99,100]}$ for the reorganization energy in diethyl ether. This would correspond to a polaron binding energy of $0.27 \mathrm{eV}$ and zero field activation energy of $0.14 \mathrm{eV}$. This is about four times larger than the reorganization energy determined by Mahrt et al. ${ }^{[101]}$ for a dispersion of TAPC in polycarbonate or in vapor-deposited layers. The zero field activation energy is only $25 \%$ of the observed activation energy, suggesting that the polaron effect is less important than the disorder effect. Combining the effect of disorder and polarons, the approximation of Equation 33 can be obtained for the zero field activation energy $\left(E_{\mathrm{a}}(E=0)\right)$.

$E_{\mathrm{a}}(E=0)=E_{\mathrm{p} / 2}+8 \sigma^{2} /(9 k T)$

According to Equation 33 an activation energy of $0.5 \mathrm{eV}$ and polaron binding energy of $0.27 \mathrm{eV}$ would yield a value of $0.10 \mathrm{eV}$ for $\sigma$. This is very close to the value of $\sigma$ obtained in the framework of the disorder model (Table 3). Application of Equation 33 suggests that even a relatively large value of $E_{\mathrm{p}} / \sigma$ will lead to a small contribution to $E_{\mathrm{a}}(E=0)$. This suggests that the effect of disorder is more important than the polaronic effect. Hence it becomes unlikely that the anomalous field, temperature, and concentration dependence of the mobility is due mainly to polaronic effects. This conclusion is supported by the similarity of the features of the transient photocurrents under conditions where the anomalous behavior is obtained to those obtained at low temperatures and high concentration.

The deviations obtained for pEFTP are probably due to the presence of several electron moieties in the same molecule. Spectro-electrochemical data ${ }^{[102]}$ suggest that in radical cations of pEFTP the hole is localized on one donor moiety. Although the data were obtained in a medium polar solvent contributing to the reorganization energy and hence favoring the localization, one may assume that this tendency will also be observed in polymer films. The Stokes shift of the fluorescence of a dilute dispersion of PEFTP in polycarbonate suggests that during the singlet lifetime relaxation of the dipolar moieties of the matrix stabilizes the dipolar excited state to some extent. ${ }^{[100]} \mathrm{A}$ similar stabilization and localization could occur during the residence time of a hole on a molecule of pEFTP dispersed in polycarbonate. Furthermore, increasing the applied field will lead to a larger free energy difference of the hole in the different triphenylamine moieties of pEFTP and hence to a more rigorous localization. This could lead to a decrease of the mobility upon increasing the applied field by a mechanism resembling the effect of off-diagonal disorder. 


\section{Conclusions}

For a large range of materials, temperatures, and applied fields, the transport of charge carriers in amorphous organic systems can be described by hopping in a Gaussian DOS. The connection between the features of the transient photocurrents and quantitative information obtained from the field and temperature dependence of the charge carrier mobility agrees with the predictions made by Monte Carlo calculations. This would suggest that for the experimentally accessible field strengths polaronic effects influence the mobility and its temperature and field dependence only to a minor extent. This has as a consequence that, when designing suitable molecules for charge transport, molecular properties determining diagonal and non-diagonal disorder are much more important than those governing electron-phonon coupling.

The deviations obtained for pEFTP can be attributed to the presence of three weakly coupled donor sites with a nitrogen-nitrogen distance of at least $12.1 \AA$. Hence even at an applied field of only $2.4 \times 10^{5} \mathrm{Vcm}^{-1}$ the energy difference between the different donor sites will be larger than $k T$ (at $298 \mathrm{~K}$ ). At an applied field of $1.1 \times 10^{6} \mathrm{Vcm}^{-1}$ this energy difference will even exceed the width of the DOS. When a more complete expression (Eq. 30 ) is used it is no longer possible to reject the polaron model on the basis of the features of the field dependence of the mobility. However, activation energies determined by a quantitative analysis of the temperature dependence of the mobility extrapolated to zero field would lead to excessive values of the reorganization energy. Comparing pEFTP to other molecules one should furthermore consider that due to the large molecular dimensions the center-to-center distance for the same concentration (in weight- $\%$ ) is significantly larger than for the smaller molecules. As, for pEFTP, deviations from the disorder model become more visible at lower concentrations, where this distance is further increased, one cannot rule out that a sufficient lowering of the concentration would, for the other transport materials, also lead to deviations from the field and temperature dependence of the charge carrier mobility predicted by the disorder model. The absence of simulations at low concentrations, however, does not allow this issue to be resolved yet.

From a fundamental point of view it is for the moment difficult to understand why Equation 16 should apparently be preferred to Equation 29, which has proved to be successful in the description of electron transfer in several liquid and solid systems. However, a closer inspection shows that when $\lambda$ is limited to values between 0.05 and $0.25 \mathrm{eV}$ the free energy dependence of the hopping rate will show a similar dependence on the free energy change for Equation 16 and Equation 28. Hence it can be expected that the mobility will show a similar field dependence. If $\lambda$ were equal to (or larger than) $0.5 \mathrm{eV}$ the mobility would increase by one order of magnitude ( $a=20 \AA$ ) when the electric field is increased from zero to $2.0 \times$ $10^{6} \mathrm{Vcm}^{-1}$. In this case a consistent interpretation of the data would no longer be possible in the framework of the disorder model. On the other hand if $\lambda$ were equal to (or smaller than) $0.025 \mathrm{eV}$ the mobility would decrease by six orders of magnitude $(a=20 \AA$ ) when the electric field is increased from zero to $1.0 \times 10^{6} \mathrm{Vcm}^{-1}$. The absence of the latter effect, in spite of the small polaron binding energies determined by Bässler, can be explained by the assumption that the electronphonon coupling is still strong enough to yield an efficient coupling to the heat bath. ${ }^{[37,103]}$ However, it cannot be excluded that the absence is due to approximations involved in the derivation of Equation 30. In this case the correct application of the polaron model would yield a field dependence of the hopping rate (Eq. 28) and the mobility that can, for all practical values of $a, \lambda$, and the applied field, not be distinguished from expressions such as Equation 16 that are based on the Miller-Abrahams expression. ${ }^{[103]}$

If polaronic effects govern charge carrier transport, the design of new transport molecules should be oriented towards relatively rigid molecules characterized by a large molecular radius, leading to a minimization of the outer sphere contribution to the reorganization energy and a minimal change of bond lengths or bond angles upon oxidation or reduction. On the other hand, if the charge carrier mobility is determined by diagonal disorder, research should be directed towards minimization of this disorder by means of suitable combinations of transport molecules and the surrounding matrix.

Received: July 7, 1993 Revised version: September 6, 1993

[1] D. M. Pai, A. R. Melnyk, Proc. SPIE 1986, 617, 82.

[2] M. A. Abkowitz, M. Stolka, in Polymers for Advanced Technologies (Ed: M. Lewin), VCH, New York 1988, p.225.

[3] D. M. Pai, B. E. Springett, Rev. Mod. Phys. 1993, 65, 163.

[4] R. H. Young, J. Appl. Phys. 1986, 60, 272.

[5] P. M. Borsenberger, W. Mey, J. Non-Cryst. Solids 1978, 28, 305.

[6] R. G. Kepler, Phys. Rev. 1960, 119, 4

[7] O. H. Le Blanc, J. Chem. Phys. 1960, 33, 626.

[8] W. E. Spear, Proc. Phys. Soc. London B 1957, 70, 669.

[9] D. J. Gibbons, A. C. Papadakis, J. Phys. Chem. Solids 1968, 29, 115.

[10] J. H. Sharp, J. Phys. Chem. 1967, 71, 2587

[11] M. Silver, M. P. Shaw, Photoconductivity and Related Phenomena (Eds: J. Mort, D. M. Pai), Elsevier, Amsterdam 1976.

[12] E. A. Silinsh, Organic Molecular Crystals, Springer Ser. Solid State Chem., Springer, Berlin 1980, p.282.

[13] A. Many, G. Rakavy, Phys. Rev. 1980, 962, 126.

[14] L. M. Schwartz, J. F. Hornig, J. Phys. Chem. Solids 1965, 26, 1821.

[15] J. R. Haynes, W. Shockley, Phys. Rev. 1951, 81, 835.

[16] R. Lawrance, A. F. Gibson, Proc. Phys. Soc. London 1952, 65B, 994.

[17] H. Scher, E. W. Montroll, Phys. Rev. B 1975, 12, 2455.

[18] A. Einstein, Ann. Phys. (Leipzig) 1905, 17, 549.

[19] B. Pollschuk, S. O. Kasap, Meas. Sci. Technol. 1991, 2, 75.

[20] F. C. Brown, F. E. Dart, Phys. Rev. 1957, 108, 281

[21] J. Lange, H. Bässler, Phys. Status Solidi b 1982, 114, 561

[22] G. Pfister, Phys. Rev. Lett. 1976, 36, 271

[23] G. Pfister, H. Scher, Adv. Phys. 1978, 27, 747.

[24] G. Pfister, H. Scher, Phys. Rev. B 1977, 15, 2062

[25] J. Noolandi, Solid State Commun. 1977, 24, 477.

[26] H. Seki, in Proc. 5th Int. Conf. on Amorphous and Liquid Semiconductors 2 (Eds: J. Stuke, W. Brenig), Taylor and Francis, London 1974, p.1015.

[27] P. Borsenberger, L. Pautmeier, H. Bässler, Phys. Rev. B 1992, 46, 12145.

[28] B. Movagahr, M. Grünewald, B. Ries, H. Bässler, D. Würtz, Phys. Rev. $B$ 1986, 33, 5545 .

[29] G. Pfister, Phys. Rev. B 1976, 16, 3676.

[30] P. M. Borsenberger, L. Pautmeier, H. Bässler, J. Chem. Phys. 1991, 95. 1258.

[31] J. M. Marshall, Rep. Prog. Phys. 1983, 46, 1235.

[32] G. Pfister, C. H. Griffith, Phys. Rev. Lett. 1978, $40,659$. 
[33] L. Pautmeier, B. Ries, R. Richert, H. Bässler, Chem. Phys. Lett. 1988, $143,459$.

[34] H. Bässler, Phys. Status Solidi b 1981, 107, 9.

[35] H. Bässler, Makromol. Chem., Macromol. Symp. 1990, 37, 1.

[36] G. Schönherr, H. Bässler, M. Silver, Phil. Mag. B 1981, 44, 47

[37] H. Bässler, Phys. Status Solidi b 1993, 175, 15.

[38] M. Silver, G. Schönherr, H. Bässler, Phys. Rev. Lett. 1982, 48, 352.

[39] P. Borsenberger, L. Pautmeier, H. Bässler, J. Chem. Phys. 1991, 94, 5447.

[40] P. Borsenberger, H. Bässler, Phys. Status Solidi b 1992, 170, 291.

[41] J. C. Scott, L. T. Pautmeier, L. B. Schein, Phys. Rev. B 1992, 46, 8603.

[42] M. Van der Auweraer, G. Verbeek, P. Borsenberger, F. C. De Schryver, unpublished.

[43] P. Borsenberger, J. Appl. Phys. 1990, 68, 5188.

[44] P. Borsenberger, J. Appl. Phys. 1990, 68, 6263.

[45] G. Verbeek, M. Van der Auweraer, F. C. De Schryver, C. Geelen, D. Terrell, S. De Meuter, Chem. Phys. Lett. 1992, 188, 85.

[46] M. Novo, M. Van der Auweraer, G. Verbeek, F. C. De Schryver, P. Borsenberger, H. Bässler, Phys. Status Solidi b 1993, 177, 223.

[47] P. Borsenberger, L. J. Rossi, J. Chem. Phys. 1992, 96, 2390.

[48] P. M. Borsenberger, Adv. Mater. Opt. Electron. 1992, $1,73$.

[49] L. B. Schein, A. Rosenberg, S. L. Rice, J. Appl. Phys. 1986, 60, 4287

[50] L. B. Schein, D. Glatz, J. C. Scott, Phys. Rev. Lett. 1990, 65, 472.

[51] D. M. Pai, J. F. Yanus, M. Stolka, D. Renfer, W. W. Limburg, Phil. Mag. $B \mathbf{1 9 8 3}, 48,505$.

[52] B. Movaghar, A. Yelon, M. Meunier, Chem. Phys. 1990, 146, 389.

[53] S. V. Novikov, A. V. Vannikov, Chem. Phys. 1993, 169, 21.

[54] L. Pautmeier, R. Richert, H. Bässler, Synth. Met. 1990, 37, 271.

[55] L. B. Schein, A. R. McGhic Phys. Rev. B 1979, 20, 1631.

[56] L. B. Schein, D. W. Brown. Mol. Cryst. Liq. Cryst. $198287,1$.

[57] K. Yokoyama, M. Yokoyama, Phil. Mag. B 1990, 61, 59.

[58] M. A. Abkowitz, H. Bässler, M. Stolka, Phil. Mag. B 1992, 63, 201.

[59] M. Gailberger, H. Bässler, Phys. Rev. B 1991, 44, 8643.

[60] P. Strohriegel, D. Haarer, Makromol. Chem., Macromol. Symp. 1991, 44, 85.

[61] W. G. Gill, J. Appl. Phys. 1972, 43, 5033.

[62] M. A. Abkowitz, J. S. Facci, W. W. Limburg, J. F. Janus, Phys. Rev. B 1992, 46, 6705

[63] M. A. Abkowitz, F. E. Knier, H.-J. Yuh, R. J. Weagley, M. Stolka, Solid State Commun. 1987, 62, 547

[64] L. Pautmeier, R. Richert, H. Bässler, Phil. Mag. B 1991, 63, 587.

[65] F. C. Bos, T. Guion, D. M. Burland, Phys. Rev. B 1989, 39, 12633.

[66] B. Ries, H. Bässler, M. Silver, Phil. Mag. B 1986, 54, 141.

[67] T. Sasakawa, T. Ikeda, S. Tazuke, J. Appl. Phys. 1989, 65, 2750.

[68] H.-J. Yuh, D. M. Pai, Mol. Cryst. Liq. Cryst. 1990, 183, 217.

[69] Y. Kanemitsu, J. Einami, Appl. Phys. Lett. 1990, 57, 673.
[70] M. Sugiuchi, H. Nishizawa, T. Uehara, Proc. 6th Int. Cong. on Advances in Non-Impact Printing Technologies, Orlando, FL, 1990 (Ed: R. J. Nash), SIST, Springfield, VA, 1991, p.298.

[71] H. Bässler, Phil. Mag. B 1984, 50, 347.

[72] T. Kitamura, M. Yokoyama, J. Appl. Phys. 1991, 69, 821

[73] A. V. Vannikov, A. Yu. Hrykov, A. G. Tyurin, T. S. Zhuravleva, Phys. Status Solidi a 1989, 115, K47.

[74] T. Holstein, Ann. Phys. (NY) 1959, 8, 253.

[75] R. A. Marcus, Ann. Rev. Phys. Chem. 1964, 13, 155.

[76] J. Jortner, J. Chem. Phys. 1976, 64, 4680.

[77] R. A. Marcus, J. Chem. Phys. 1984, 81, 4494.

[78] L. B. Schein, Phil. Mag. B 1992, 65, 795

[79] Y. Taniguch, T. Fujiyama, H. Tanaka, M. Yokoyama, Solid State Commun. 1991, 80, 817.

[80] P. Borsenberger, H. Bässler, J. Chem. Phys. 1991, 95, 5327.

[81] K. M. Weibel, H. Bässler, J. Chem. Phys. 1986, 84, 1590.

[82] P. Borsenberger, Adv. Mater. Opt. Electron. 1992, $1,73$.

[83] P. M. Borsenberger, J. Phys. Chem. 1993, 91, 4815.

[84] A. Peled, L. B. Schein, Chem. Phys. Lett. 1988, 153, 422.

[85] D. Haarer, H. Schörer, A. Blumen, in Dynamical Processes in Condensed Molecular Systems (Eds: J. Klafter, J. Jortner, A. Blumen), World Scientific, Singapore 1989 , p.107.

[86] M. Van der Auweraer, F. C. De Schryver, P. Borsenberger, unpublished.

[87] S. Facci, M. Stolka, Phil. Mag. B 1986, 54, 1 .

[88] P. Borsenberger, J. lmaging Sci. 1991, 35, 79.

[89] P. M. Borsenberger, J. Appl. Phys. 1990, 68, 4100.

[90] D. Emin, Phys. Rev. B 1993, 46, 9419.

[91] M. Abkowitz, D. Rice, M. Stolka, Phil. Mag. B 1990, 61, 25.

[92] Y. Yamaguchi, M. Yokoyama, J. Appl. Phys. 1991, 70, 3726.

[93] E. A. Silinsh, G. A. Shlihata, A. J. Jurgis, Chem. Phys. 1991, 155, 389.

[94] J. C. Scott, L. Pautmeier, L. B. Schein, Chem. Phys. Lett. 1992, 197, 568.

[95] M. R. V. Sahyun, Photogr. Sci. Eng. 1984, 28, 185.

[96] L. B. Schein, D. Glatz, J. C. Scott, Phys. Rev. Lett. 1990, 65, 42.

[97] G. Verbeek, A. Vaes, M. Van der Auweraer, F. C. De Schryver, C. Geelen, Macromolecules 1993, 26, 472.

[98] B. S. Brunschwig, S. Ehrenson, N. Sutin, J. Phvs. Chem. 1987, 91, 4714.

[99] G. Verbeek, S. Depaemelaere, M. Van der Auweraer, F. C. De Schryver, A. Vaes, D. Terrell, S. De Meutter, Chem. Phys. 1993, $176,195$.

[100] M. Van der Auweraer, F. C. De Schryver, G. Verbeek, A. Vaes, N. Helsen, P. Van Haver, S. Depaemelaere, D. Terrell, S. De Meutter, Pure Appl. Chem. 1993, 65, 1665.

[101] R. I. Mahrt, J. Yang, H. Bässler, Chem. Phys. Lett. 1992, 192, 576.

[102] J. Bonvoisin J.-P. Launay, M. Van der Auweraer, F. C. De Schryver, unpublished.

[103] A. Miller, E. Abrahams, Phys. Rev. 1960, 120, 745. 